\title{
Contributi per uno studio sulla sismicità dell'Italia
}

\author{
L. Mintelit - P. MoNTecciII
}

Ricevuto il 28 IIarzo 1962

\section{INTRODLZIONE E GENERALITA.}

E noto che quando si parla di "sismicità " della terra in generale, o in particolare di una regione, non si intende definire in maniera precisa un concetto fisico o geofisico, ma piuttosto si vuole esprimere una maggiore o minore "attitudine" di una determinata regione ad essere sede di fenomeni sismici.

Per lo più la sismicità, così com'è intesa generalmente comprende un panorama vasto e piuttosto disordinato di un insieme di coefficienti che nella loro totalità danno l'idea del fenomeno senza per altro dare al fenomeno stesso un preciso significato fisico, esprimibile in termini matematici. Essa è una funzione della distribuzione geografica dei terremoti, della loro frequenza, della loro intensità, della loro energia, della loro natura, della loro correlazione con elementi geologici, e cosi via. I qui la grande difficoltà di dare una definizione sintetica di tut to ciò.

La cosa più semplice e più lineare per affrontare lo studio della sismicità di una regione, è ovviamente quella di documentarsi il più possibile su tutti i terremoti avrenuti in tale regione; localizzarne gli epicentri in modo da averne una distribuzione geografica; poi, con l'ausilio delle nozioni fornite dalla sismologia, ricercare tutti quegli elementi che servono a valutare l'intensità e l'energia di ogni singola scossa: sfruttare infine i risultati ottenuti per dare un aspetto organico a tutto il fenomeno nel suo insieme.

Un lavoro di questo genere, esteso a tutta la terra, servirebbe a dare una visione esatta della sismicità globale.

La sismologia però è una scienza tanto giovane che non ha a sua disposizione un numero così esteso (nello spazio e nel tempo) di dati di osservazione tali da consentire una trattazione esauriente dell'argomento. Per quanto la installazione di numerose stazioni sismiche, disseminate in vari punti del globo, consenta di " captare " la maggior parte 
dei movinenti della terra, pure arviene che tutti quei piccoli terremoti che sviluppano una scarsa energia e che hamo il loro epicentro in zone disabitate, o sfornite di osservatori, sfuggono al rontrollo generale; sirchè può arcadere, come difatti arviene, che le carte sismiche di certe rescioni presentino una concentrazione di epicentri superiore a quella di altré, ma ciò unicamente a causa di una diversa distribuzione delle stazioni sismiche, o di attrezzature strumentali diverse, e non già perchè questo corrisponda ad una effettiva situazione locale.

Per quanto riguarda poi il periodo che precede lavrento della sismologia, intesa come scienza applicata allo studio dei fenomeni geofisici, le notizie riguardanti i terremoti hammo il sapore della cronaca e occorre andare a documentarsi su carteggi locali, rhe sovente sono incompleti o alterati, o mancano addirittura.

Da tutto ciò lisultano chiare le difficoltà inerenti ad un tale problema inteso nella sua estensione.

E noto come vari tentativi per magiungere un accordo su un metodo comume per la stima della sismicità siano stati via via scartati per la loro insufficienza (cosi il metodo basato semplicemente sul numero delle scosse, oppure quello basato sulla intensità massima valutata macrosismicamente, a cosi pure quello rhe tiene conto della frequenzal,... exe.).

Al momento attuale si tende a calcolare la sismicità di una regione in base all'energia totale liberata per unità di superficie durante una determinata unità di tempo. Questa quantità, corredata con i dati che asprimono il numero, la frequenza e la intensità dei sismi, sembra finora. essere uno dei modi più esatti per esprimere quantitativamente, con pieno significato fisico, utilizzabile per ulteriori sviluppi, le caratteristiche sismiche di una determinata area.

Tuttavia per quanto vari siano i tentativi e le proposte per definire in termini matematici ma grandeza cosi complessa come quella rella sismicità, non si è ancom giunti al un accordo internazionale che consenta una definizione adottata da tutti.

Nei vari congressi dell'U.G.G.I. (Stocearda 1952; Roma 1954; Viemna 1956; Utrecht 1958; Alicante 1959; Helsinki 1960) fu maturato dalla Commissione Sismologica Europea un programma di ricerca inteso a tale scopo e furono invitate le nazioni Europece facenti parte di tale commissione a compilare delle mappe sismiche (riferentesi al periodo 1901-1955) complete degli elementi essenziali (data del sisma, ora, coord. geografiche epicentrali, profondità ipocentrale, intensità macrosismica. all'epicentro, raggio della isosista di Vo) in modo da renderle il più omoge:ere possibili. 
Quando sarà completata ma mappa sismica europea, omogenea secondo lo spirito dei congressi su ritati, si samà compiuto indubbiamente un passo avanti per uno studio sistematico della sismica dell'Europa. In questi studi però verramo a mancare i contributi apportati dalle scosse meno intense, crenendo prese in considerazione solamente le $I_{o} \geqslant V I^{\circ}$ ) e se ciò può essere inessenziale in un quadro d'insieme non si dere tuttavia, a nostro arriso, sottovalutame limportanza ai fini di una panolamica regionale.

\section{LA SINAICITA IX BASE ALL'FXERGA.}

Poichè è nostra intenzione portare un contributo allo studio della sismicità dell'Ttalia con metodi il più rigorosi possibile, riteniamo che il modo migliore sia, secondo le più recenti tendenze, appunto quello di legare i fenomeni sismici alle valutazioni della magnitudo, per risalire poi da questa al calcolo dell'energia erogata in un dato periodo.

Da quando nel 1935 Richter introdusse il concetto di Magnitudo, un g an passo aranti fu compiuto nella serie delle scale macrosismiche, togliendo alle valutazioni delle intensità ogni carattere arbitrario (dovuto a tanti coefficienti diversi), dando così una interpretazione eschusiramente strumentale.

is noto come anche il Gutenberg si sia appassionato all'argomento e ne abbia fatto, insieme col Richter, oggetto di studi sempre più estesi e sempre più approfonditi tanto da imporlo all'attenzione di tutto il mondo della sismologia, talchè si può dire che oggi quasi tutti gli osservatori riportino nei loro rilievi sismici anche i dati delle mannitudo calcolate nelle singole stazioni.

Eे proprio alla magnitudo che si può ricorrere per poter clare al problema che ci interessa un arattere obbiettivo di dati strumentali.

Sono note le relazioni che intercorrono tra questa grandezar e l'energia sismica.

Riportiamo qui le ultime due date da Richter e Gutenberg nel 1956 per la Califomia, e quella calcolata per l'Italia. Esse somo:

$$
\begin{aligned}
& \log E=9,4+2,14 M-0,054 M-(\text { Gutenberg-Richter) (1956ia) } \\
& \log E=11,8+1,5 M \quad \text { (Gutenberg-Richter) (1956b) } \\
& \log E=9,154+2,147 \text { II (Di Filippo-Marcelli) (1950). }
\end{aligned}
$$

X. B. - M. Bath nel 1956 da la seguente relaziono

$$
\log E=12.24-1.44 . M
$$

che hen si accorda con la seconda delle tre. 
Se quindi, come si è detto, adottiamo per la valutazione della sismicita il concetto dell'energia totale liberata per unità di superficie, durante una determinata unità di tempo, è ovvio che il problema si impernia tutto sul calcolo delle magnitudo delle singole scosse.

E necessario a questo punto osservare che il valore di $M$ è essenzialmente strumentale e che le considerazioni su esposte verrebbero a mancare in tutti quei casi in cui i numerosissinni terremoti di piecola intensità che avvengono in tutte le regioni della terra, non avessero un'energia sufficiente a propagare le onde sismiche fino alle più prossime stazioni di registrazione.

Si presenta quindi la necessitì di trovare un metodo che consenta di calcolare la magnitudo anche a prescindere dalle registrazioni strumentali e ciò si può ottenere imponendo un legame tra la intensità macrosismica della scossa all'epicentro e la sua magnitudo.

Oecorre cercare cioè una relazione che possa essere espuressa come funzione di $I_{0}$ erl $M$.

Osserviamo ulteriormente che anche questa considerazione può fallire per quei terremoti che avessero il loro epicentro in zone disabitate o nelle aree marine, per le quali mancherebbero i dati necessari alla ralutazione secondo la Scala Mercalli-Sieberg; però dato che noi ci accingiamo ad impostare il problema nei rignardi dell Italia, che è una regione densamente popolata, risulterà in definitiva che molto pochi saramno $\mathrm{i}$ terremoti che sfuggiramo al controllo e comunque saranno certamente tali da non influenzare, con la loro assenza, il quadro genemale della sismicitì.

\section{Ricerca Dellat FUxzIoNe: $f\left(I_{o}, M\right)$.}

La risoluzione di questo problema la imposteremo su un metodo matematico a carattere statistico; ci serviremo di dati sicuri, accuratamente rilevati dalle registmaioni ottenute nella stazione sismica di Roma, per un elevato numero di terremoti vicini e domestici, di cui si conoscono le intensità epicentrali.

Supporremo inizialmente che la funzione $f\left(I_{0}, M\right)$ da noi cercata sia del tipo

$$
\mathrm{I}=m \mathrm{I}+c
$$

e conoscendo i valori di $\mathrm{I}$ e $Y$, che sono rispettivamente la magnitudo $M$ 
e l'intensità $T_{0}$ allepicentro, le nostre due incognite diventano i due roefficienti numerici $m$ e c. La nostra equazione sarà quindi

$$
H=I_{0} y+x
$$

Allo scopo di poterei servire di dati di partenza (.1I ed $I_{0}$ ) il piu precisi possibile, abbiamo ritenuto opportumo valerei prevalentemente di uno studio elfettuato dal De Panfilis, il quale con analisi minuziosa ed accurata ricopre i rilievi maxposismici di tutto il territorio italiano nel periodo 1953-5T. Questo studio ei ha consentito di servirci di numerosi valori di $I_{0}$ accumatamente valutati.

Poichè della maggior parte di questi terremoti averamo, come si è detto, disponibili le registrazioni della stazione sismiea di Roma, abbiamo potuto calcolare con sufficiente esattezza la magnitudo di 59 terremoti, dei quali, nella tabella $I^{a}$, riportiano i dati rilevati.

Oltre che di questi elementi ci siamo serviti anche dei dati di altri terremoti che per motivi vari furono precedentemente fatti oggetto di particolare attenzione e di studi accurati. In questo secondo gruppo (le rui date esulano dal periodo 1953-57) abbiamo incluso anche 8 terremoti con epicentro nella zona di Terni (Cmbria), appartenenti al periodo sismico che per vari mesi, a partire dal Iuglio 1960, ha interessato la zona stessa.

Il numero dei dati cosi utilizzati è risultato complessivamente di 83 terremoti, come risulta dalla tabella $I^{\mathrm{a}}$. Notiamo a questo punto che tutti gli elementi di cui abbiamo potuto cosi usufuire si riferiscono a terremoti di intensità piuttosto moderata (raramente l'intensità all'epicentro ha raggiunto l'VIIIo).

Fortumatamente in Italia quei cataclismi, che scomvolgono altre regioni della terma, sono piuttosto rari. Parossismi di notevole rilievo si sono avuti in tempi storici, ma la preponderanza assoluta attuale per lo meno nel secolo corrente - è data dai gradi non superiori al VIVII Mercalli. Tuttavia, allo scopo di usuf ruire anche di qualche elemento che, pur raro, può pur sempre verificarsi, abbiamo ritenuto opportuno inserire nel calcolo anche $\mathrm{i}$ due terremoti disastrosi del 1908 (Messina) e del 1915 (Avezzano), le cui magnitudo sono state calcolate da Gutenberg.

Il metodo dei minimi quadrati, che qui applichiamo, porta a risolvere com'è noto, per ma serie di equazioni del tipo

$$
a_{i} x+b_{i} y=c_{i}
$$

un sistema della forma seguente:

$$
\left\{\begin{array}{l}
{[a a] \cdot x+[a b] y=[a c]} \\
{[b a] \cdot b+[b b] y=[b c] .}
\end{array}\right.
$$


Tenenclo conto che nel nostro aso dalle [1'] si ha che

$$
a_{i}=1 ; b_{i}=I_{0} ; c_{i}=I I
$$

i valori dei coefficienti risultano, a conti fatti, i seguenti:

$$
\begin{array}{lll}
{[a a]=\Sigma a_{i}=85} & {[b a]=\Sigma\left(I_{0}\right)_{i}} & =492 \\
{[a b]=\Sigma\left(I_{0}\right)_{i}=492} & {[b b]=\Sigma\left(I_{0}^{2}\right)_{i}} & =2981 \\
{[a c]=\Sigma(M)_{i}=356,075} & {[b c]=\Sigma\left(1 I I_{0}\right)_{i}} & =2125,07
\end{array}
$$

sicehè tutto si riduce a risolvere il sistema:

$$
\left\{\begin{array}{r}
85 x+492 y=350,075 \\
492 x+2981 y=2125,07 .
\end{array}\right.
$$

I)i qui si traggono, per le nostre incognite, i valori:

$$
\left\{\begin{array}{l}
x=1,4070 \\
y=0,4806
\end{array}\right.
$$

sicchè la relazione rercata assume la forma:

$$
M^{*}=0,4 \$ 1 I_{0}+1,40 \%\left({ }^{(1)}\right. \text {. }
$$

Questa funzione risponde soddisfarentemente allo scopo. Infatti, sostituendo in essa i valori delle intensità e delle magnitudo di numerosi terremoti aceuratamente documentati, per le intensità comprese dal III" al VII0-VIII Mercalli-Sieberg si sono trovati risultati pienamente attendibili. Tale approssimazione non è però altrettanto soddisfacente per i gradi superiori, per i quali invere la funzione presenta una certa tendenza a perdere il suo calattere di linearità.

Tuttaria questo inconveniente non toglie valore al significato dellespressione da noi trovata, giacehe la massima frequenza dei sismi italiani si rerifica proprio per quelli di $I_{0} \leqslant$ VIII.

Per gli eventuali terremoti di grado superiore all'VIII non sarebbe necessario ricorrere a tale espressione, poichè la nostra rete di stazioni sismiche, abbastanza fita, darebbe ottine registrazioni capaci di fornire dati strumentali ben precisi.

Tonostante queste lecite considerazioni, abbiamo voluto spingere oltre l'indagine anche in vista dell'utilità che potrà avere una siffatta relazione per la conoscenza in temini di $I$ di tutti quei terremoti storici di cui sono state tramandate soltanto notizie macrosismiche.

(1) X.B. Indichiano con .17 la magnitudo calcolata dalle registrazioni e scentizil di $l_{0}$. (o) $M^{*} n$ " $"$ attraverso lat cono- 
Dalla posizione sul grafico dei punti $\left(L_{a}, M\right)$ per i gradi più elerati, abbiamo pertanto ritenuto opportuno supporre, per la $j\left(I_{0}, I\right)$ un andamento parabolico. In tal senso abbiamo seritto la funzione nella forma:

$$
y=\alpha r^{2}+\beta x+\gamma .
$$

Abbiamo cosi dovuto lisolvere un sistema di s:) equazioni (nelle 3 incognite $\alpha, \beta, \gamma)$, del tipo:

$$
M=\alpha I_{0}{ }^{2}+\beta I_{o}+\gamma .
$$

Anche qui abbiamo applianto il metodo dei minimi qualdati che. nel caso in esame, conduce a risolvere un sistema della forma seguente:

essenclo.

$$
\begin{aligned}
{[a a] c e+[a b] \beta+[a c] \gamma } & =[a l] \\
{[b b \cdot 1] \beta+[b c \cdot 1] \gamma } & =[b l \cdot 1] \\
{[c c \cdot 2] \gamma } & =[c l \cdot 2]
\end{aligned}
$$

$$
a_{i}=I_{a}=, b_{i}=I_{a}, c_{i}=1, l_{i}=M .
$$

La seguente tabella IIa riporta i risultati dei calcoli per la determinazione dei coefficienti.

'T'abolla II

$$
\begin{aligned}
& \text { [na] } \cdots \sum\left(I_{0}^{2}\right)^{2}-128332,250 \quad[b b]=\Sigma\left(I_{0}\right)^{2}=2981,000 \\
& \mid a b] !=\Sigma\left(I_{0}^{2}\right) \cdot\left(I_{0}\right)=19005,000 \quad[b c]=\Sigma I_{0}=492,000 \\
& \text { |ac }]=\Sigma\left(I_{0}{ }^{2}\right) \cdot(1)=2981,000 \quad[b l]=\Sigma I_{0} . H=2130,950 \\
& {[a l]=\Sigma\left(I_{0}^{2}\right) \cdot . l=13423,7+5} \\
& \begin{array}{l}
{[c r]= \pm 1^{2}=85,000} \\
{[r l]=\Sigma(1, M)=356,060}
\end{array} \\
& {[b b \cdot 1]=[b b]-\frac{[a b]}{[a a]}[a b]=168,260} \\
& {[b c \cdot 1]=[b c]-\frac{\lfloor a b]}{[a a]}[a c]=50,812} \\
& {[b l \cdot 1]=[b l]-\frac{[a b]}{[a a]}[a l]=1+4,236} \\
& {[c c \cdot 1]=[c c]-\frac{[a c]}{[a a]}[a c]=1, \bar{s}, 8+1} \\
& {[c l \cdot 1]=[c l]-\frac{[a c]}{[a a]}[a l]=4+, 629} \\
& \begin{array}{l}
{[c \cdot 2]-[c c \cdot 1]-\frac{\mid l \cdot c \cdot 1]}{[b b \cdot 1]}[b \cdot \cdot 1]=0.496} \\
{[c l \cdot 2]=[c l \cdot 1]-\frac{[b \cdot-1]}{[0 \dot{b} \cdot 1]}[b l \cdot 1]==1.070}
\end{array}
\end{aligned}
$$


Liequazione alla quale siamo infine pervenuti, è la seguente

$$
U^{*}=0,00+I_{0}+0,206 I_{o}+2,153
$$

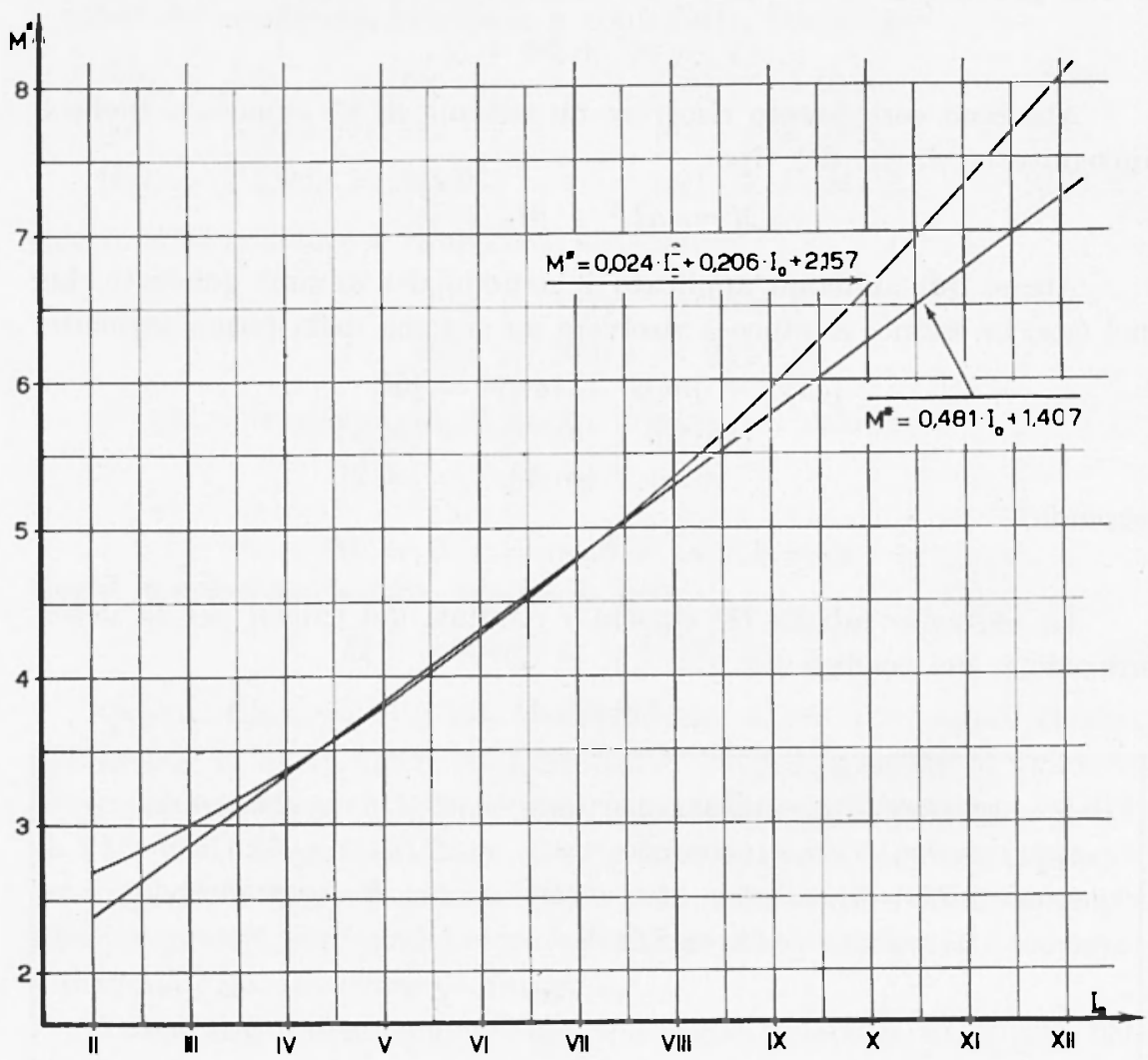

Firg. 1

Nella Fig. 1 sono riportate in grafico le due equazioni [2] e [3]. E notevole osservare come la retta e la parabola abbiano un lungo tratto in culi praticamente coincidono. Ciò avviene fin verso il VIIo-VIIIo della scala Mercalli-Sieberg. Riteniamo quindi senz'altro lecito servirci indifferentemente dell'una o dell'altra fino a tale valore, mentre per i gradi superiori si rende necessario il solo uso della curva del II0 ordine.

\section{FATTORI CORRETTIYT REGIONALI.}

È evidente che una relazione, che leghi la magnitudo con l'intensità macrosismica allepicentro, puó variare da regione a regione. 
Questo viene già messo in evidenza da tutte le relazioni trovate finora dai vari autori:

Per la California Gutenber'ge e Richter danno (1956):

$$
\left.M=2 / 3 I_{0}+1 \quad \text { (per } h=16-18 \mathrm{~km}\right) .
$$

Per la regione del Jura Souabe, Peterschmitt nel 1950 areva dato:

$$
\left.M=0,8 I_{v}-0,9 \quad \text { (per } h=10-15 \mathrm{~km}\right) \text {. }
$$

Per la regione del Caucaso, Sararenshy e I)zibladze trovano (nel $1950(i)$

$$
M=0,69 I_{0}+0,9 .
$$

Per le montagne dei Carpazi (nel 1958) Shebalin dà:

$$
\begin{cases}M=0,7 I_{0}+2,3 \log h-2,0 & {[\text { per } 0<h<60-100 \mathrm{~km}]} \\ M=0,7 I_{0}+2,3 \log h-3,6 & {[\text { per } h>60-100 \mathrm{~km}] .}\end{cases}
$$

Per la Cina, nel 1958, Lee trova:

$$
M=0,58 I_{0}+1,5 .
$$

Per la Cecoslovacehia, Kalmik (1960) dà:

$$
M=0,67 I_{0}+1,7 \log h+1,+1 .
$$

Per la Grecia, Galanopoulos (1962) dà:

$$
M=\vartheta+0,38(\vartheta-6,0)
$$

essendo $\theta=\log A+\log I_{0}$ (dove $A=$ area macrosismica in kn²). (Ed altri ancoral).

Per quello che riguarda l'Italia in particolare possiamo ritenere che le formule da noi trovate vadano bene per tutti i terremoti di profonclità nomale $(h<20 \mathrm{~km})$, come sono genemblnente quelli whe arvengono nella alea italiana, ad eccezione di alcuni sismi profondi, localizzati tuttavia nelle zone vulcaniche, prevalentemente del basso Tirreno e per i quali si potrà eventualmente fare ma considerazione a parte.

La nostra stessa formula [2], che è stata licavata, come si è detto, da un insieme di dati rignardanti un certo numero di epicentri clisseminati sull'intero territorio nazionale, va fatta oggetto di ma considerazione particolare. La magnitudo, rilevata tramite la conoscenza di $I_{o}$, trattandosi di terremoti vicini, è strettamente legata alle fenomenologie seologiche dei luoghi inrestiti dalle onde sismiche, specialmente per i gradi più bassi. Abbiamo quindi ritenuto opportuno proveredere alla ricerca di eventuali fattori correttivi da inserire nella formula trovata, plopli delle varie regioni geologiche italiane. 
Per questo seopo ci siamo ealeolate, con la [2] le varie magnitudo in base alle intensita epicentrali secondo la scala Morcalli, quindi, rimiti i parossismi per regioni, abbiamo computato lo scarto di ogni terremoto trata magnitudo $M$ oftenuta dalla rempistmazione strumentale e quellat $\left(I^{*}\right)$ attribuibile in base alla funzione $f\left(I_{0}, M\right)$. Gli scarti $\varepsilon$ (riportati mell'ultima colomma della tab. I) sono stati mediati, secondo un (riterio regionale, ot temendo in tal modo i cercati fattori correttivi, che riportiamo quil di seguito:

1) Regione Alpina (con particolare riguardo alla parte orientale) $=-0,37$

2) Rogrone dell'Enilia a Romagna $=+0,31$

3) Regione Toseana $=-0,17$

1) Regione Appenninica e Adriatica dell'Italia (entrale $=-0,20$

5) Regione Abruzzese e Campana dell'Appennino $=+0,01$

b) Regione Pugliese, Incana e della Calabria Settentrionale = - 0,02

7) Regrome ('alabrese o dei massicei cristallini (compresa la parte N. flella Sicilial $)=+0,0-4$

8) Per la regrone Siciliana a per duella Tirrenica del Lazio e della Campania, non disponendo di dati sufficienti, non è stato possibile stabilire il compispondente fattore correttion.

L'allegata carta d'Italia mette in evidenza i risultati conseguiti. I fattori correttivi regionali, circoscritti da linee continue rendono anevole l'uso della carta, della quale di si potrà servire ogni qualvolta si voglia calcolare la magniturlo di un terremoto di coli si conosea l'intensità epirent rale e l'epicentro macrosismicos.

La [2] assumeria quindi l'aspetto delinitivo:

$$
M^{*}=0,+\$ 1 I_{0}+1,40 \%+1 \equiv .
$$

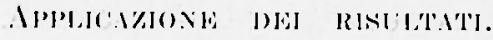

C'ome applicazione dei risultati ottenuti ci serviremo di essi per fare III esame il più completo possibile della sismicità dell'Italia per il periodo 1953-57. In esso, conformemente allo spirito dei congressi dell'U.G.G.I. furono dal De Panfilis prese in particolare esame le scosse di $I_{0} \geqslant V^{0}$; peró nella sua pubblicazione latutore non trascura aleuni elementi essenziali che consentono mat visione generale del fenomeno sismico nel 
I. MARCELLI - P. MONTECCHI

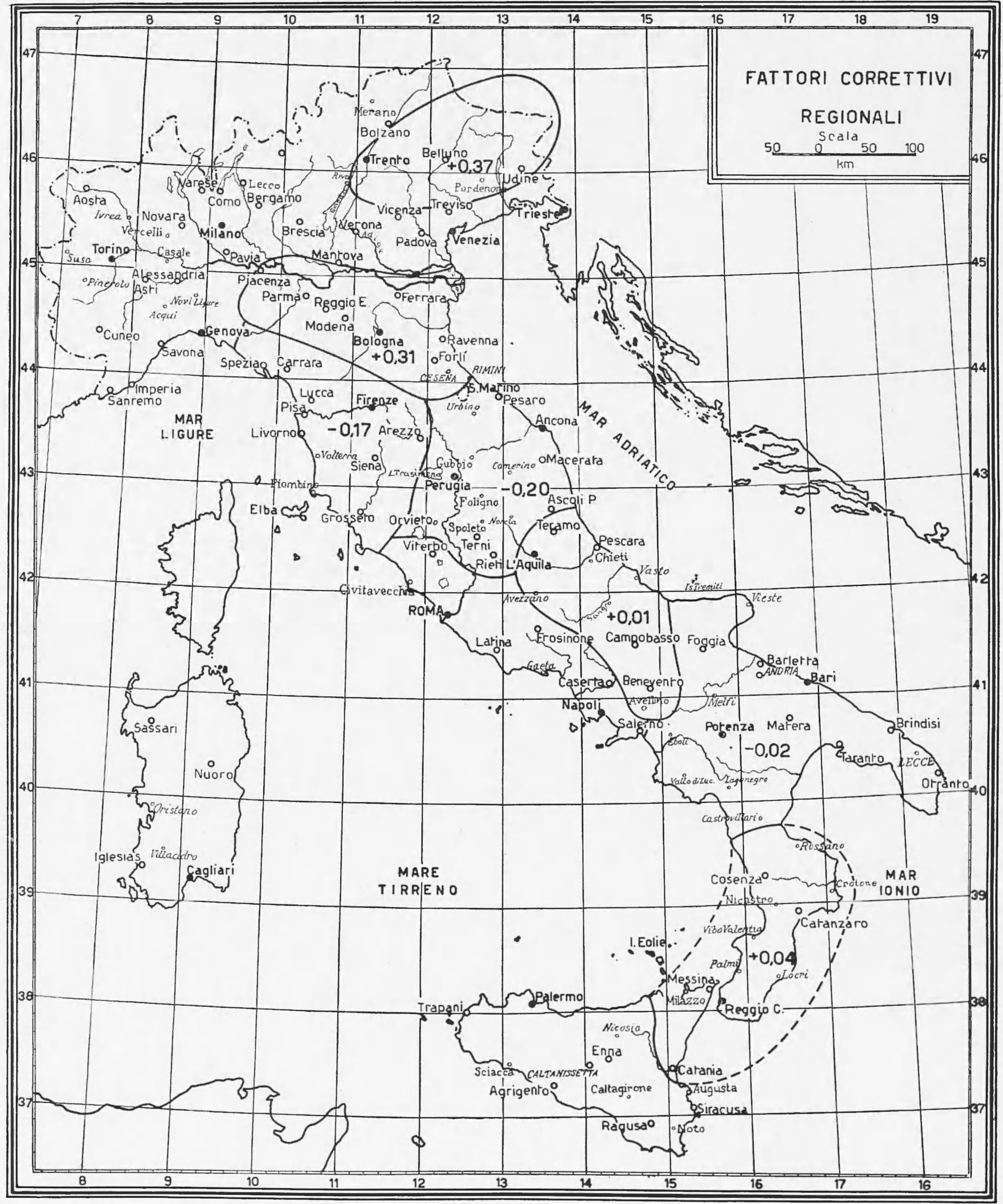


quinquennio; abbiamo potuto cosi in un primo tempo computare in percentuale il numero delle scosse dei vari gradi verificatesi durante il periodo predetto: risulta cosi che il

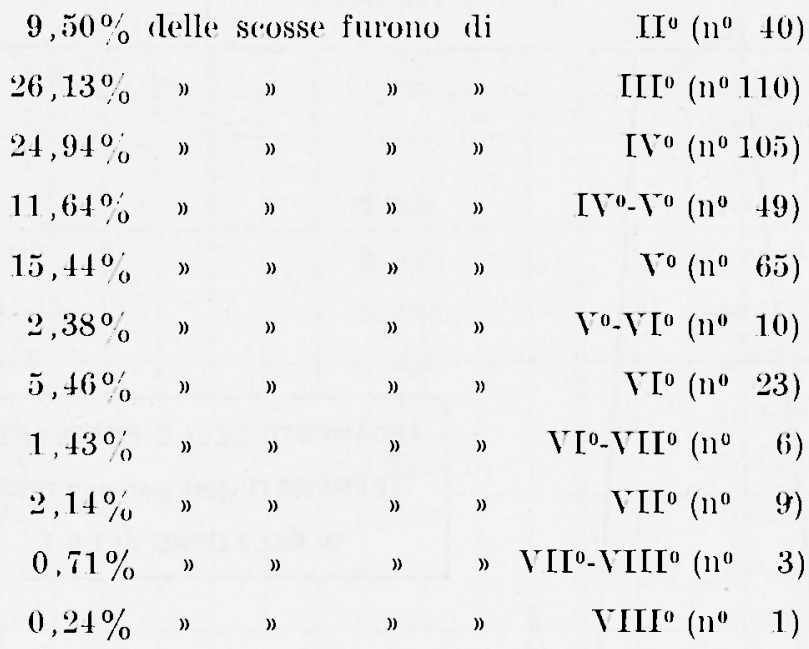

Il gratico della Fig. 2 riassume in forma più evidente le caratteristiche salienti.

Si nota una preponderanza assoluta di piccole scosse comprese tra il III0 e il IVo, mentre i gradi superiori al $V^{0}$ - per il quale c’è ancora una percentuale piuttosto elevata - vamno diminuendo sensibilmente di frequenza. Già questo quadro, nella sua visione sommaria mette in evidenza il arattere di moderata sismicità che compete all'Italia, per lo meno per quanto si riferisce al periodo in esame. Alle scosse più sopra schematicamente elencate, e che sono quelle ben determinate rispetto all'epicentro, al tempo ed all'intensiti, se ne debbono aggiungere numerose altre le cui notizie vaghe ed inprecise non consentono una ulreriore precisazione: tutte però di lieve entità e principalmente repliche dei terremoti più importanti.

Agli eftetti di un calcolo dellenergia sismica totule liberata, i terremoti di grado inferiore al $V^{0}$ contribuiscono con $u$ apporto di energia molto minore rispetto a quella fornita anche da un solo terremoto di grado V. Allo scopo di chiarire tale concetto in termini più evidenti, tenendo presente la $[z]$ e la relazione di Gutenborry-Richter.

$$
\log E=11,8 \div 1,5 M
$$




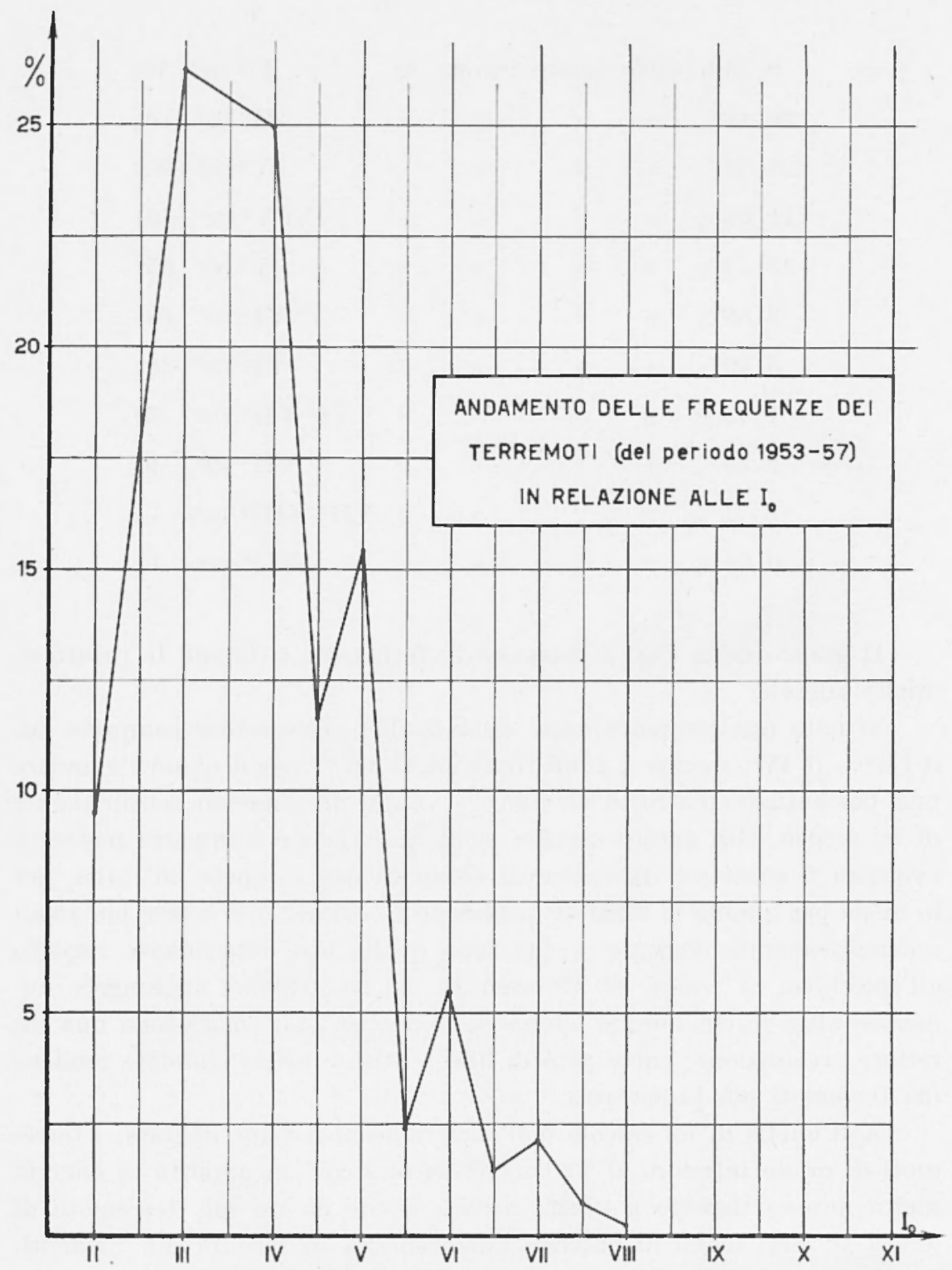

F'ig. 2 
abbiamo calcolato la seguente tabella III (e relativo grafico della Fig. 3) nei quali vengono poste in corrispondenza le grandezze $I_{n}, I^{*}$, ed $E$.

Tabel!a III

\begin{tabular}{|c|c|c|}
\hline$I_{o}$ &.$L^{*}$ & 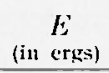 \\
\hline II & 2,369 & $10)^{15,353}$ \\
\hline III & 2,850 & $10^{16,075}$ \\
\hline III-IV & 3,090 & $10^{16,435}$ \\
\hline IV & 3,331 & $10^{16,796}$ \\
\hline$I V-Y$ & 3,572 & 1017.158 \\
\hline V & 3,812 & $10^{17,518}$ \\
\hline $\mathrm{V} \cdot \mathrm{VI}$ & 4,053 & $10^{17,879}$ \\
\hline VI & 4,293 & $10^{18,2611}$ \\
\hline VI-VII & 4,534 & 1018,601 \\
\hline VII & 4,775 & $10^{18,9633}$ \\
\hline VII-VIII & 5,052 & $10^{19,378}$ \\
\hline VIII & 5,341 & $10^{19,812}$ \\
\hline VIII-IX & 5,642 & $10^{20,263}$ \\
\hline$I X$ & $5,95 \overline{5}$ & 1()$^{20,733}$ \\
\hline$I X \cdot X$ & 6,280 & $10^{21,220}$ \\
\hline $\mathrm{X}$ & 6,617 & $10^{21,726}$ \\
\hline $\mathrm{X} \cdot \mathrm{XI}$ & 6,9665 & $1(1)^{22,249}$ \\
\hline XI & 7,327 & 1()$^{22.791}$ \\
\hline XI-XII & 7.700 & $100^{23,350}$ \\
\hline XII & 8,085 & I(1)23, \\
\hline
\end{tabular}

Dalla tabella risulta abbastanza chiaramente che il passaggio da ciaseun grado Merealli al suecessivo equivale ad una erogazione di energia multipla della precedente secondo un fattore quasi costantemente uguale a $10^{0,7}$ fino al grado VII, ed un po' maggiore dal grado VII in poi; sicche, a titolo di esempio si può dire che un solo terremoto di grado $\mathrm{V}$ equivale a quasi 30 terremoti di grado III; o anche che per sviluppare l'energia di un terremoto di VIo occorrerebbero circa 150 scosse di 
grado IIT. Tale ronsiderazione giustificherebbe parzialmente la decisione adottata dalla commissione sismologica Europea di tener conto solo delle scosse di grado $\geqslant$ VI. Il trascurare le inferiori non costituisce,

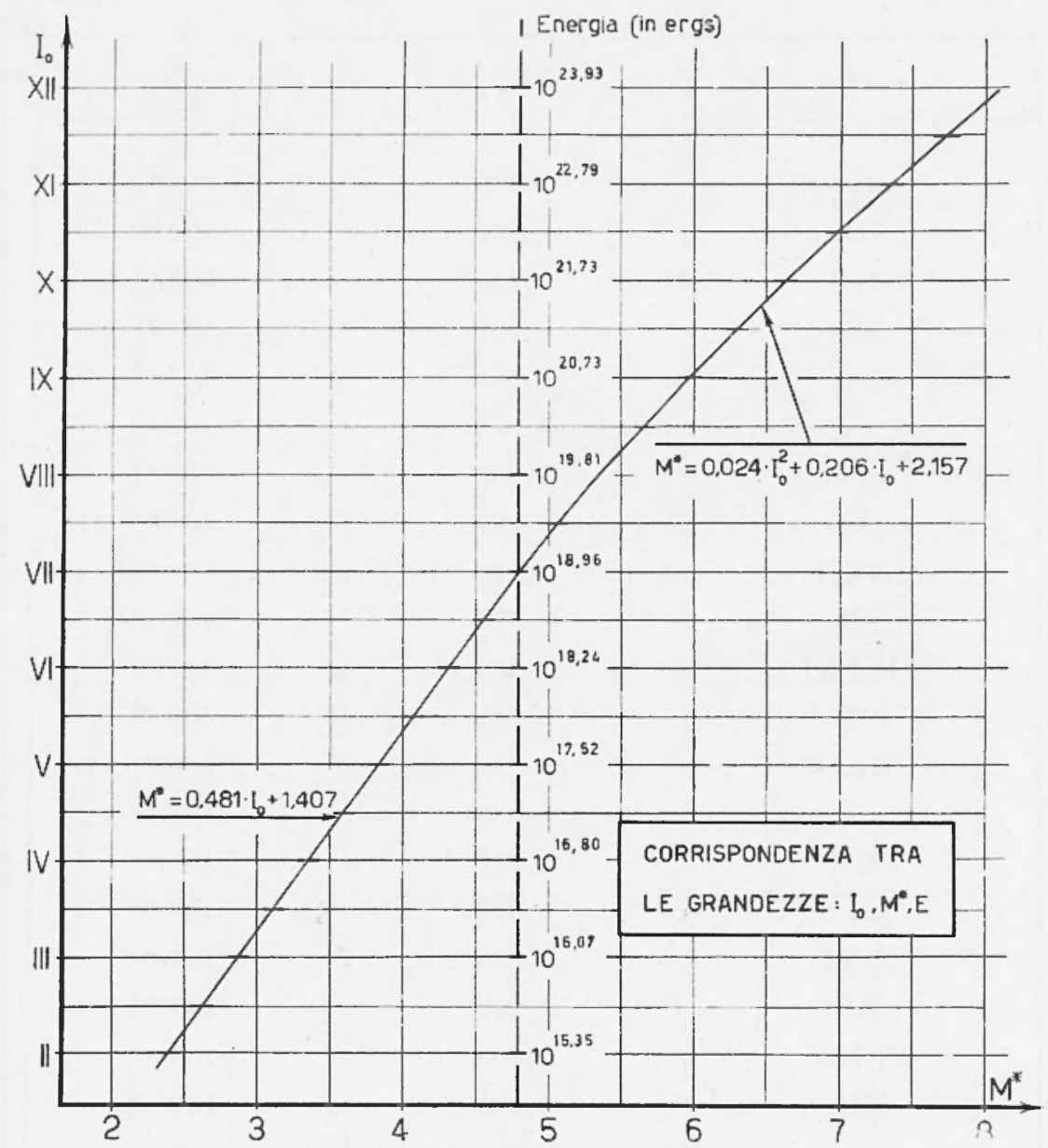

Fig. 3

in effetti, una deficienza sostanziale nel panomma sismico generale specialmente dal punto di vista energetico. Però, a nostro arviso, e come abbiamo precedentemente affermato, ai fini di una panoramica regionale, riteniamo che non sia troppo giusto sottovalutare l'importanza di queste scosse, che sovente sono l'espressione di una graduale liberazione di energie accumulatesi negli strati più superficiali della terra. 
Tella tabella IV sono riportati i risultati dei calcoli delle energie che competono ad ogni terremoto del quinquennio in esame. Per le scosse la cui magnitudo non si era potuta prelevare dalle registrazioni ci siamo serviti della [ $\left.2^{\prime}\right]$ conoscendo la $I_{n}$ : e per le ragioni sopra esposte, nel romputo dellenergia abbiamo incluso anche quei terremoti di grado inferiore al $\mathrm{V}$, di cui il De Panfilis non dà indicazioni particolareggiate, limitandosi a dime il numero e la distribuzione nelle varie classi ed intensita. Il numero di questi ultimi compare entro parentesi nella tabella $\mathrm{V}$, la quale riassume i risultati oftenuti.

Tell'ultima coloma di questa tabella è riportato il contributo dei singoli anni all'energia sismi(“a (1), precisando però che esso si riferisce a tutti quei terremoti le cui magnitudo sono state calcolate singolarmente (v. tabella IV), e nell'ultima riga compaiono le energie complessive relative ai singoli gradi. (Questi ultimi dati sono stati calcolati per via esclusivamente teorica applicando la [2] e la [3]. L'energia dovuta ai 141 terremoti studiati è dunque $=10^{15} \cdot 1177561,5943$ ergs $=10^{-1} \cdot 1,1776$.

Per avere l'energia complessiva del quinquennio occorre però, come si è detto, tener conto anche dei contributi dei gradi inferiori: e precisa mente:

10 scosse di II contribuiscono per $10^{15} \cdot 6,9512 \quad$ ergs

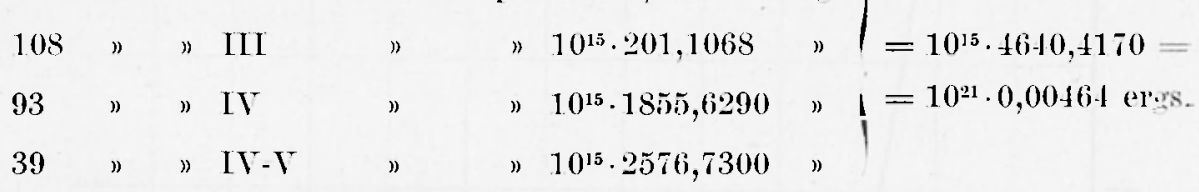

Sicchè in definitiva possiamo dire che l'energia sismica erogata sul territorio nazionale durante gli anni 1953-5̃ è di

$$
E=10^{15} \cdot 1.182 .202,0113=1,1822 \cdot 10^{21} \mathrm{ergs}
$$

pari ad una media ammule di circa

$$
E_{\text {annuale }}=0,24 \cdot 10^{21} \mathrm{erg} ; .
$$

11 grafico della Fig. $t$ riassume efficacemente la situazione sismica del quinquennio in esame. $\mathbf{E}$ ivi messo in chiam evidenza lo scarso con-

(1) Per questa applicazione, nel computo delle energie, abbiamo usato la relazione $\log E=9,154+2.1+7.11$ (Di Filiplo-Marcelli) che per $\mathrm{i}$ gradi $\leqslant$ Vll concorda abbastanza con quella di Gutenberg. I risultati conseguiti, tuttavia, risultano sopravalutati rispetto a quelli che si otterrebbero applicando la formula di Gutenberg (1956b). 
tributo di energia apportato dalle scosse di grado inferiore al VI: non è però altrettanto trascurabile la loro frequenza che si presenta invece piuttosto elevata.

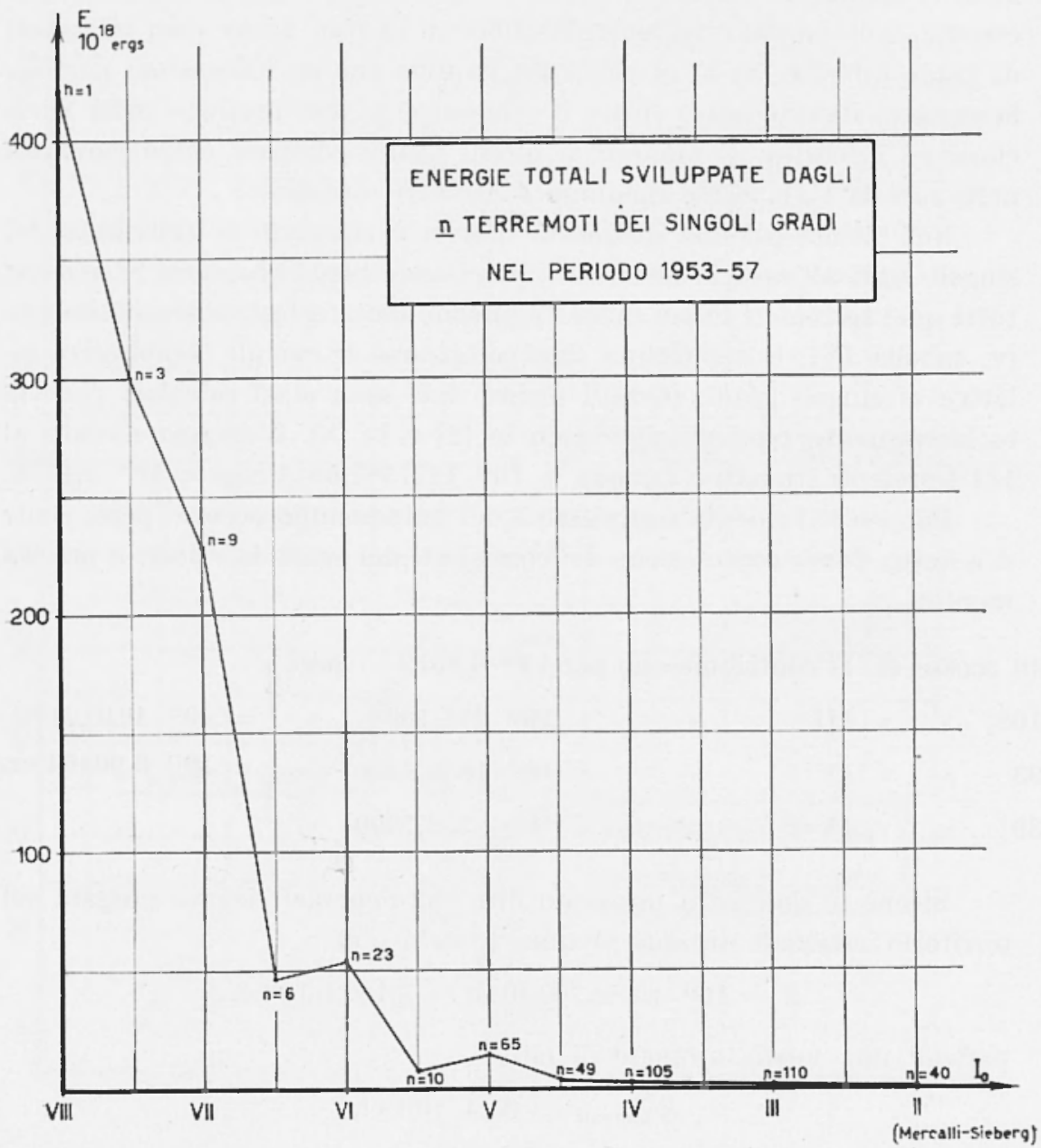

Fig. 4

Non è tuttavia lecito, da questi risultati parziali, trarre conclusioni di carattere generale, anzitutto perchè 5 anni, nella storia sismica di una regione, costituiscono una parte infinitesima, e poi perchè l'intervallo di tempo studiato si è presentato particolarmente tranquillo rispetto ad altri periodi. Se soltanto rolessimo tener presenti i terremoti di Messina 
(del 1908) e quello di Avezzano (del 1915) - per non parlare che dei più tristemente noti - vedremmo come questa media potrebbe aumentare considerevolmente.

Abbiamo infine compilato una ulteriore tabella (la VI) nella quale compaiono 45 terremoti scelti tra quelli più dettagliatamente studiati e per i quali sono state calcolate le aree macrosismiche e i raggi medi delle varie isosiste cosi come possono essere state desunte dal lavoro citato. I tentativi fatti per trovare una qualche relazione matematica tra questi elementi e le altre grandezze specifiche di ogni terremoto $\left(I_{0}, M, E,\right)-$ cosi come è stato fatto da vari autori in altre parti della terra - sono tutti falliti; ma cio non deve stupire se si pensa che la configurazione geologica del suolo italiano varia continuamente da un punto all'altro e che pertanto gli effetti macrosismici di ogni scuotimento, essendo intimamente legati alla natura degli strati terrestri più superficiali (oltre che alla profondità ipocentrale) non possono avere alcun carattere di omogeneità. Tutt'al più si può riscontrare un ripetersi di caratteristiche per le scosse che provengono, in tempi diversi, dagli stessi epicentri.

Roma - Istituto Nazionale di Geofisica - Marzo 1962. 


\begin{tabular}{|c|c|c|c|c|c|c|c|c|c|c|c|}
\hline$\underset{\infty}{\stackrel{*}{*}}$ & $\begin{array}{l}0 \\
0 \\
+\end{array}$ & $\begin{array}{l} \pm \\
0 \\
1\end{array}$ & $\begin{array}{l}01 \\
0 \\
+\end{array}$ & $\begin{array}{l}\hat{\theta} \\
0 \\
+\end{array}$ & $\begin{array}{l}+ \\
0 \\
0 \\
+\end{array}$ & $\begin{array}{l}\overrightarrow{1} \\
0 \\
1\end{array}$ & $\begin{array}{l}\stackrel{i}{+} \\
0 \\
1\end{array}$ & $\begin{array}{l} \pm \\
0 \\
+\end{array}$ & $\begin{array}{l}\mathfrak{a} \\
0 \\
+\end{array}$ & $\begin{array}{l} \pm \\
0 \\
1\end{array}$ & $\begin{array}{l}0 \\
\infty \\
0 \\
+ \\
+\end{array}$ \\
\hline 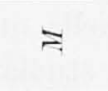 & $\begin{array}{l}\stackrel{0}{+} \\
+\end{array}$ & $\begin{array}{l}0 \\
\oplus \\
\oplus\end{array}$ & $\begin{array}{l}\stackrel{9}{+} \\
\dot{7}\end{array}$ & $\begin{array}{l}\stackrel{P}{i} \\
\dot{+}\end{array}$ & $\stackrel{10}{=}$ & एँ & $\vec{H}$ & $\begin{array}{l}10 \\
\infty \\
\infty\end{array}$ & $\begin{array}{l}\mathscr{0} \\
\dot{+}\end{array}$ & $\stackrel{10}{\rightarrow+}$ & $\begin{array}{l}\dddot{\theta} \\
+\end{array}$ \\
\hline$-i$ & 5 & $>$ & $>$ & $D$ & $>$ & $\Rightarrow$ & $>$ & $>$ & $>$ & $\triangleright$ & $>$ \\
\hline 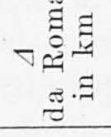 & $\stackrel{8}{\stackrel{8}{+}}$ & \& & $\begin{array}{l}0 \\
\infty \\
\text { o }\end{array}$ & 年 & $\frac{\vec{d}}{\infty}$ & $\stackrel{8}{\underline{I}}$ & 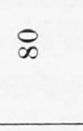 & ন্ & $\vec{\sigma}$ & $\begin{array}{l}8 \\
\text { 践 }\end{array}$ & $\stackrel{\text { to }}{\mathscr{\sigma}}$ \\
\hline 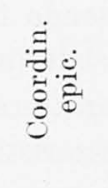 & 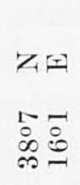 & 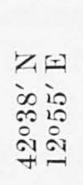 & 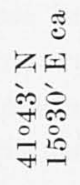 & $\begin{array}{l}z=1 \\
100 \\
00 \\
\dot{0}=0\end{array}$ & 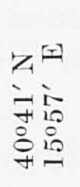 & 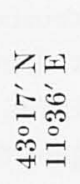 & 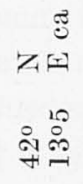 & 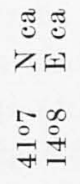 & 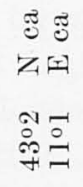 & 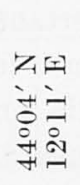 & 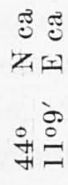 \\
\hline $\begin{array}{l}\stackrel{0}{0} \\
0 \\
\vdots \\
z \\
0 \\
0 \\
0 \\
= \\
0 \\
0 \\
0 \\
\approx \\
0 \\
\therefore\end{array}$ & 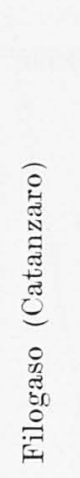 & 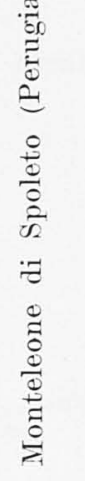 & 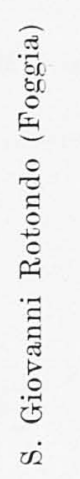 & 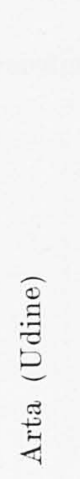 & 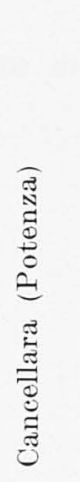 & 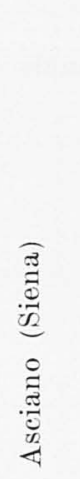 & 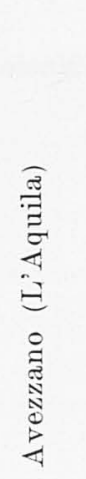 & 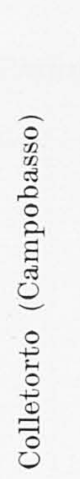 & 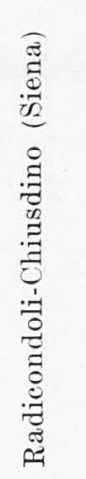 & 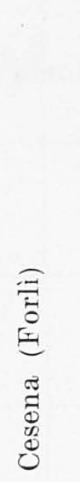 & 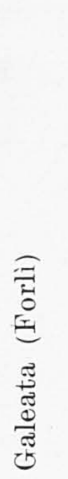 \\
\hline is & $\mathscr{8}$ & 10 & $\stackrel{0}{f}$ & 18 & $\stackrel{\theta}{2}$ & 10 & $\int$ & $\stackrel{\infty}{\infty}$ & $\stackrel{\text { I }}{\sim}$ & $\stackrel{8}{0}$ & $\stackrel{\theta}{2}$ \\
\hline శ & to & $\vec{\infty}$ & $\stackrel{\leftrightarrow}{*}$ & $\vec{\infty}$ & $\stackrel{\infty}{0}$ & คิ & $\stackrel{9}{\sim}$ & 18 & is & $\exists$ & $\mathscr{8}$ \\
\hline$=$ & 8 & $\stackrel{\infty}{\circ}$ & $\stackrel{\theta}{-2}$ & จิ & $\vec{b}$ & $\stackrel{0}{-1}$ & t' & $\mathscr{8}$ & $\stackrel{\circ}{\sim}$ & $\stackrel{\infty}{\circ}$ & $\mathscr{8}$ \\
\hline \multirow{3}{*}{$\begin{array}{l}\pi \\
+ \\
\pi \\
0\end{array}$} & $\begin{array}{l}\text { i⿱ } \\
0 \\
-2\end{array}$ & $=$ & $=$ & $=$ & $=$ & $=$ & $=$ & $=$ & $=$ & $=$ & 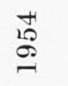 \\
\hline & $\begin{array}{l}\stackrel{0}{\pi} \\
\frac{1}{6} \\
0 \\
0 \\
0 \\
4\end{array}$ & $=$ & 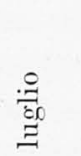 & $\hat{=}$ & $\begin{array}{l}0 \\
\pm \\
0 \\
0 \\
+0 \\
0\end{array}$ & $=$ & 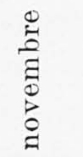 & 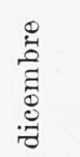 & $=$ & $=$ & $\begin{array}{l}\stackrel{\Omega}{\mathbb{N}} \\
\stackrel{\Xi}{\Xi}\end{array}$ \\
\hline & ลอ & $\stackrel{\circ}{\sim}$ & $\stackrel{\oplus}{\sim}$ & $\stackrel{10}{\mathrm{~N}}$ & 20 & $\stackrel{\theta}{\sim}$ & హ & $\leftrightarrow$ & r & $\stackrel{\leftrightarrow}{-}$ & ㅇ \\
\hline z & - & or & $\infty$ & $H$ & 10 & $\infty$ & 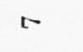 & $\infty$ & 0 & $\stackrel{-}{\sim}$ & $\Xi$ \\
\hline
\end{tabular}




\begin{tabular}{|c|c|c|c|c|c|c|c|c|c|c|c|}
\hline$\stackrel{\vec{l}}{i}$ & $\begin{array}{l}a \\
0 \\
+\end{array}$ & $\begin{array}{l}0 \\
01 \\
0 \\
+\end{array}$ & $\begin{array}{l}\infty \\
+ \\
0 \\
+\end{array}$ & $\begin{array}{l}\infty \\
0 \\
0 \\
+\end{array}$ & $\begin{array}{l}0 \\
0 \\
1\end{array}$ & $\begin{array}{l}2 \\
0 \\
0 \\
1\end{array}$ & $\begin{array}{l}9 \\
0 \\
+\end{array}$ & $\begin{array}{l}\infty \\
0 \\
0 \\
+\end{array}$ & $\begin{array}{l}0 \\
0 \\
0 \\
1\end{array}$ & $\begin{array}{l}12 \\
0 \\
+\end{array}$ & $\begin{array}{l}0 \\
0 \\
0\end{array}$ \\
\hline$z$ & $\begin{array}{l}\overline{6} \\
\overline{7}\end{array}$ & $\begin{array}{l}10 \\
+9 \\
\end{array}$ & $\begin{array}{l}\mathrm{F} \\
\mathrm{H}\end{array}$ & $\begin{array}{l}\text { ro } \\
+\end{array}$ & $\begin{array}{l}t \\
\dot{t}\end{array}$ & $\begin{array}{l}5 \\
\mathrm{a}\end{array}$ & $\frac{0}{10}$ & $\stackrel{0}{\rightarrow}$ & $\begin{array}{l}\dddot{b} \\
\infty\end{array}$ & $\begin{array}{l}\vec{f} \\
\dot{f}\end{array}$ & $\begin{array}{l}\Re \\
\stackrel{-}{-}\end{array}$ \\
\hline- & $\bar{\nabla}$ & $\bar{D}$ & $\overline{5}$ & $>$ & $\Xi$ & 5 & $\stackrel{\Xi}{\leftrightarrows}$ & $>$ & $\overline{7}$ & 5 & 5 \\
\hline 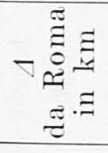 & $\bigotimes_{10}$ & $\frac{10}{\infty}$ & $\frac{0}{10}$ & ‡ & 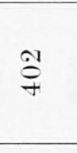 & $\begin{array}{l}10 \\
\infty \\
\text { a }\end{array}$ & $\begin{array}{l}10 \\
10 \\
01\end{array}$ & $\underset{\mathrm{S}}{\mathrm{S}}$ & $\begin{array}{l}10 \\
\infty \\
-1\end{array}$ & $\stackrel{10}{12}$ & $\stackrel{\text { जे }}{+}$ \\
\hline 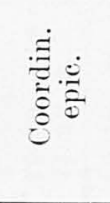 & 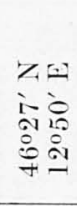 & 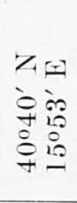 & $\begin{array}{l}z=1 \\
0 \\
0 \\
0 \\
0 \\
0 \\
0 \\
0\end{array}$ & 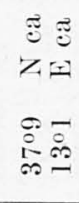 & 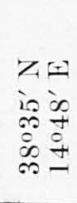 & 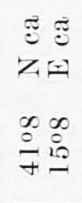 & 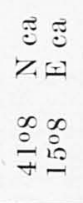 & 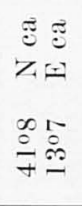 & $\begin{array}{rl} & 8 \\
Z & \\
\infty & \infty \\
0 & \infty \\
0 & 0\end{array}$ & 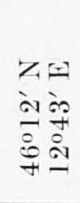 & 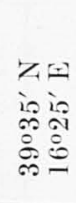 \\
\hline $\begin{array}{l}0 \\
0 \\
\Xi \\
\pm \\
0 \\
0 \\
0 \\
0 \\
0 \\
\approx \\
0 \\
\approx\end{array}$ & 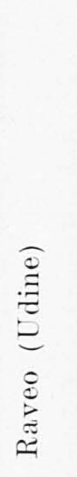 & $\begin{array}{l}\frac{\pi}{0} \\
\stackrel{0}{0} \\
0 \\
0 \\
0\end{array}$ & 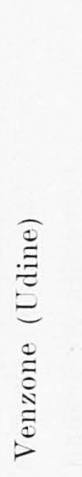 & 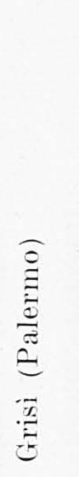 & 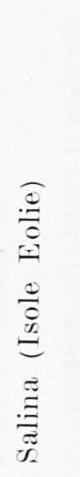 & 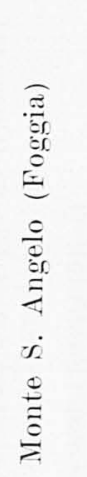 & $\begin{array}{l}= \\
=\end{array}$ & 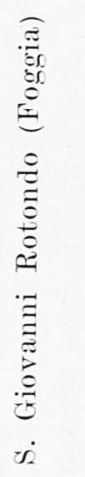 & 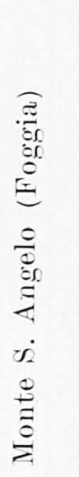 & 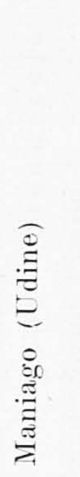 & 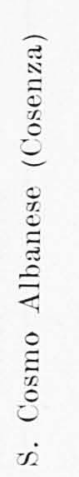 \\
\hline $\begin{array}{l}\infty \\
\Xi \\
=\end{array}$ & $\begin{array}{l}\frac{\sigma}{2} \\
\stackrel{2}{a}\end{array}$ & $\begin{array}{l}\frac{1}{2} \\
\frac{\pi}{9} \\
\stackrel{1}{1}\end{array}$ & $\begin{array}{l}10 \\
91 \\
18 \\
4 \\
12\end{array}$ & $\begin{array}{l}5 \\
5 \\
5 \\
=\end{array}$ & $\begin{array}{l}10 \\
10 \\
10 \\
10 \\
0 \\
0\end{array}$ & $\begin{array}{l}\dot{0} \\
\infty \\
\infty \\
\infty\end{array}$ & $\begin{array}{l}5 \\
\infty \\
0 \\
+ \\
5\end{array}$ & $\begin{array}{l}\tilde{5} \\
\sigma \\
\dot{+} \\
8 \\
0\end{array}$ & 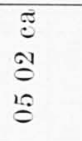 & $\begin{array}{l}\overrightarrow{0} \\
15 \\
0 \\
0\end{array}$ & 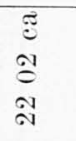 \\
\hline \begin{tabular}{l}
$\pi$ \\
\multirow{\pi}{*}{} \\
$\tilde{\sigma}$
\end{tabular} & $\begin{array}{l}\frac{0}{\vec{\Xi}} \\
\frac{\pi}{3} \\
10 \\
\text { oi }\end{array}$ & 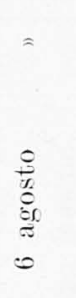 & $\begin{array}{l}\frac{0}{5} \\
\frac{0}{0} \\
\frac{1}{0} \\
= \\
=\end{array}$ & 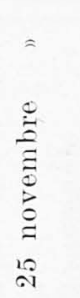 & 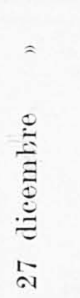 & 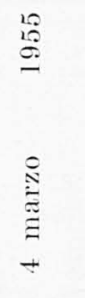 & $\stackrel{\infty}{\infty}$ & 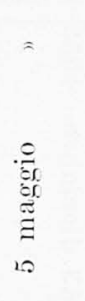 & $\begin{array}{l}\stackrel{0}{\stackrel{0}{\Xi}} \\
\stackrel{\curvearrowright}{=}\end{array}$ & $\begin{array}{l}\hat{\sigma} \\
\ddot{i}\end{array}$ & $\approx$ \\
\hline$z$ & $\stackrel{N}{\sim}$ & $\stackrel{\Re}{-}$ & \pm & $\stackrel{20}{2}$ & 0 & 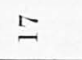 & $\underline{-}$ & $\stackrel{\theta}{-}$ & ลे & $\vec{\sigma}$ & I \\
\hline
\end{tabular}




\begin{tabular}{|c|c|c|c|c|c|c|c|c|c|c|c|}
\hline$\stackrel{*}{=}$ & $\begin{array}{l}8 \\
0 \\
+ \\
+\end{array}$ & $\begin{array}{l}\ddot{\infty} \\
0 \\
1\end{array}$ & $\begin{array}{l}\hat{\theta} \\
\dot{\theta} \\
1\end{array}$ & $\begin{array}{l}\tilde{0} \\
0 \\
+\end{array}$ & $\begin{array}{l}8 \\
0 \\
+\end{array}$ & $\begin{array}{l}= \\
0 \\
1\end{array}$ & $\begin{array}{l}\ddot{0} \\
\dot{0} \\
+\end{array}$ & $\begin{array}{l}\infty \\
0 \\
0 \\
+\end{array}$ & $\begin{array}{l}10 \\
0 \\
1\end{array}$ & $\begin{array}{l}\overrightarrow{0} \\
0 \\
+\end{array}$ & $\begin{array}{l}\overline{0} \\
0\end{array}$ \\
\hline$=$ & $\begin{array}{l}\ddot{\infty} \\
\infty\end{array}$ & $\mp$ & $\begin{array}{l}\stackrel{8}{1} \\
\infty\end{array}$ & $\stackrel{\infty}{+}$ & $\begin{array}{l}90 \\
0 \\
+\end{array}$ & $\infty$ & $\frac{9}{20}$ & $\begin{array}{l}\theta \\
\infty \\
\infty\end{array}$ & $\begin{array}{l}\infty \\
\infty \\
\infty\end{array}$ & $\begin{array}{l}\dddot{\vartheta} \\
-\end{array}$ & $\begin{array}{l}\stackrel{1}{9} \\
\leftrightarrow\end{array}$ \\
\hline$-i$ & - & $\Xi$ & $>$ & $>$ & $\overline{7}$ & $=$ & $\Xi$ & $>$ & 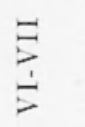 & $\equiv$ & $=$ \\
\hline 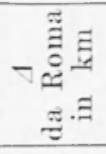 & 点 & $\stackrel{\leftrightarrow}{\circ}$ & $\stackrel{1}{ \pm}$ & $\stackrel{19}{9}$ & 志 & $\underset{\mathrm{N}}{\mathrm{S}}$ & $\stackrel{\leftrightarrow}{\circ}$ & $\S$ & $\underset{\text { 壱 }}{+}$ & $\stackrel{\circ}{\circ}$ & $\stackrel{\mathrm{ai}}{-}$ \\
\hline 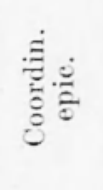 & 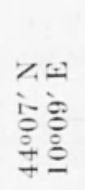 & 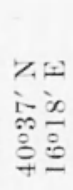 & 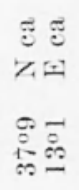 & 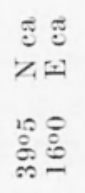 & 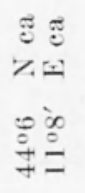 & $\begin{array}{l}z=1 \\
\text { ò } \\
0 \\
\dot{0}\end{array}$ & 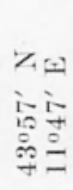 & 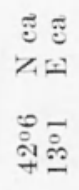 & 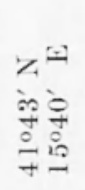 & 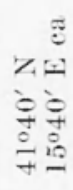 & 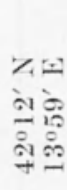 \\
\hline 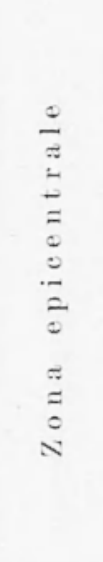 & 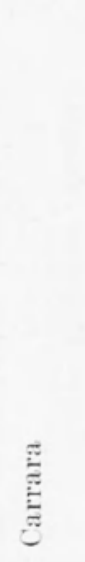 & 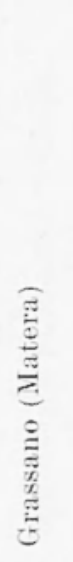 & 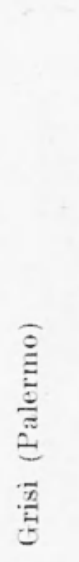 & 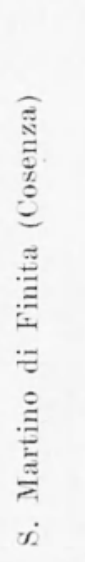 & 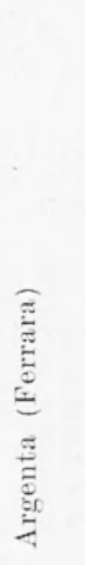 & 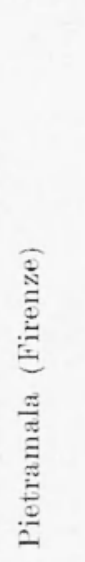 & 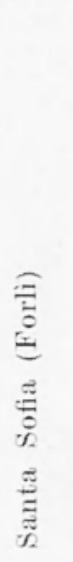 & 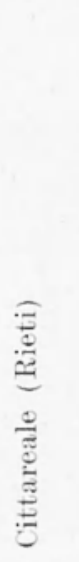 & 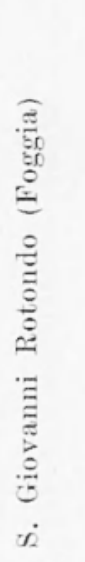 & $\begin{array}{l}= \\
= \\
=\end{array}$ & 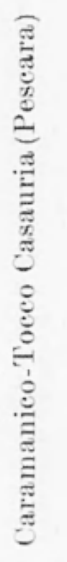 \\
\hline 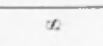 & 5 & $\mathbb{E}$ & $\tilde{B}$ & $\tilde{G}$ & $\widetilde{3}$ & $\dddot{\sigma}$ & $\widetilde{3}$ & $\tilde{\mathfrak{J}}$ & $\mathbb{T}$ & $\stackrel{g}{9}$ & 7 \\
\hline$\Xi$ & $\vec{b}$ & $\mp$ & $\underline{\theta}$ & ลิ & $\overrightarrow{\mathrm{i}}$ & 8 & $q$ & 30 & $\stackrel{\leftrightarrow}{\circ}$ & $\theta$ & $\vec{d}$ \\
\hline$\Rightarrow$ & $\stackrel{\infty}{2}$ & $\bar{\sigma}$ & $\stackrel{\leftrightarrow}{\circ}$ & $\mathscr{8}$ & $\stackrel{9}{6}$ & $\vec{b}$ & $\stackrel{9}{2}$ & $\stackrel{8}{8}$ & $\cong$ & $\Xi$ & $\bar{\sigma}$ \\
\hline & $\begin{array}{l}10 \\
\stackrel{10}{9}\end{array}$ & 苞 & $=$ & $=$ & $=$ & $=$ & $\approx$ & $=$ & $=$ & $=$ & $=$ \\
\hline $\begin{array}{l}\ddot{\pi} \\
\ddot{\pi} \\
\ddot{0}\end{array}$ & 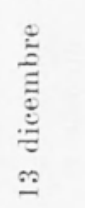 & 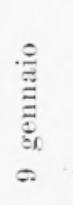 & 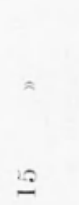 & $\begin{array}{l}\Rightarrow \\
\mathrm{s}\end{array}$ & 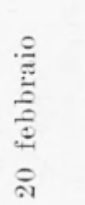 & 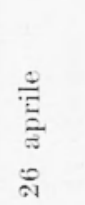 & 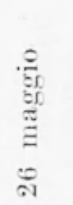 & 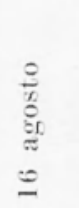 & $\begin{array}{l}= \\
\pm\end{array}$ & 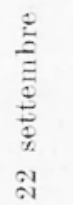 & $\begin{array}{l}\frac{0}{3} \\
\frac{3}{0} \\
0 \\
0 \\
0\end{array}$ \\
\hline$z$ & $\ddot{\leftrightarrow}$ & $\vec{\Delta}$ & 10 & จิ & to & $\stackrel{\infty}{\rightarrow}$ & $\overrightarrow{\mathrm{i}}$ & $\stackrel{8}{0}$ & $\vec{\infty}$ & $\stackrel{9}{\therefore}$ & $\dddot{\leftrightarrow}$ \\
\hline
\end{tabular}




\begin{tabular}{|c|c|c|c|c|c|c|c|c|c|c|c|}
\hline$\stackrel{*}{=}$ & $\begin{array}{l}5 \\
0 \\
+\end{array}$ & $\begin{array}{l}\dot{8} \\
\dot{0}\end{array}$ & $\stackrel{9}{1}$ & $\begin{array}{l}9 \\
0 \\
0\end{array}$ & $\begin{array}{l} \pm \\
0 \\
+\end{array}$ & $\stackrel{i}{i}$ & $\overrightarrow{0}$ & $\stackrel{9}{\circ}$ & $=$ & $\begin{array}{l}13 \\
0 \\
+\end{array}$ & $\begin{array}{l}5 \\
0 \\
1\end{array}$ \\
\hline$\Rightarrow$ & $\begin{array}{l}\stackrel{0}{0} \\
-\end{array}$ & $\begin{array}{l}\stackrel{-1}{-} \\
-\end{array}$ & $\begin{array}{l}\overrightarrow{0} \\
\infty\end{array}$ & $\begin{array}{l}\dddot{\theta} \\
+\end{array}$ & $\stackrel{\leftrightarrow}{-}$ & $\begin{array}{l}\text { 它 } \\
\therefore 0\end{array}$ & $\begin{array}{l}0 \\
\therefore\end{array}$ & $\begin{array}{l} \pm \\
+\end{array}$ & $\frac{\stackrel{N}{-}}{+}$ & $\begin{array}{l}\infty \\
\infty \\
\infty\end{array}$ & $\begin{array}{l}\mathrm{r} \\
\stackrel{5}{*}\end{array}$ \\
\hline-2 & $\Xi$ & 5 & $>$ & 5 & $=$ & - & 5 & $\Xi$ & 5 & $>$ & $>$ \\
\hline 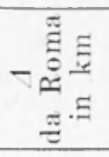 & $\frac{19}{10}$ & $\stackrel{\infty}{=}$ & $\stackrel{\mathrm{N}}{-}$ & $\S$ & $\stackrel{\oplus}{\mathscr{0}}$ & $\stackrel{9}{-}$ & 39 & î & $\underset{\sim}{\stackrel{1}{1}}$ & 兽 & $\stackrel{8}{\varnothing}$ \\
\hline$\underset{\dot{\Xi}}{\dot{\Xi}}$ & 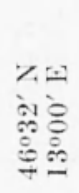 & 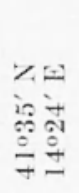 & 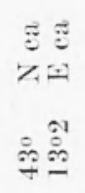 & 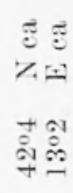 & 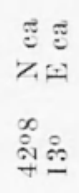 & 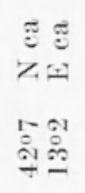 & 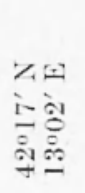 & $\begin{array}{l}210 \\
+0 \\
10 \\
0 \\
90 \\
9\end{array}$ & 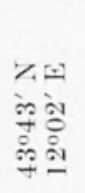 & 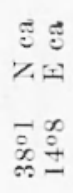 & 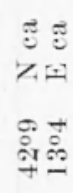 \\
\hline 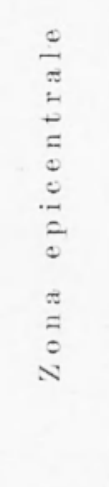 & 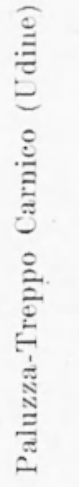 & 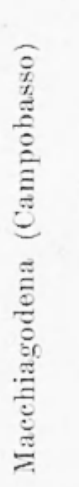 & 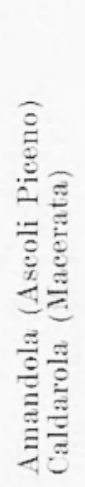 & 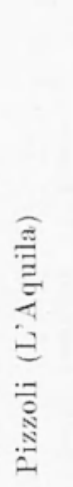 & 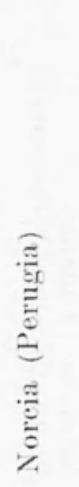 & 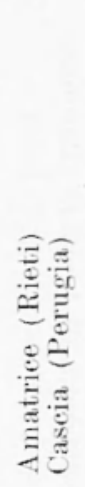 & 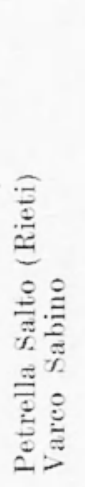 & 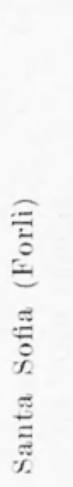 & 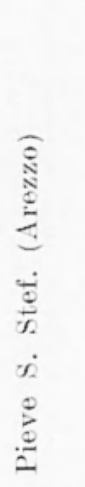 & 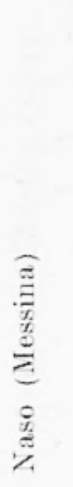 & 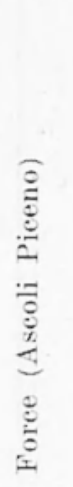 \\
\hline$x$ & बे & $q$ & $\widetilde{3}$ & $\tilde{3}$ & $\infty$ & שु & 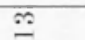 & $\stackrel{\circ}{\oplus}$ & $\stackrel{\infty}{0}$ & $\mathscr{T}$ & $\mathbb{8}$ \\
\hline$\Xi$ & 19 & $\ddot{g}$ & $\ddot{i}$ & 9 & $\theta$ & $\cong$ & $\underline{\theta}$ & 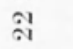 & 19 & \pm & i \\
\hline$=$ & ลิ & $\dddot{\ominus}$ & $\vec{\sigma}$ & $\stackrel{9}{6}$ & \pm & $\overrightarrow{0}$ & 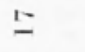 & $\dddot{\sigma}$ & 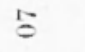 & $\vec{b}$ & 워 \\
\hline & 范 & $=$ & $=$ & 点 & $=$ & $=$ & $=$ & $=$ & $=$ & $=$ & $\approx$ \\
\hline 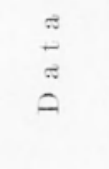 & 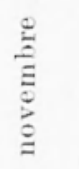 & $=$ & $=$ & 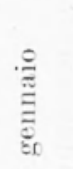 & 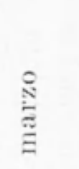 & $\approx$ & 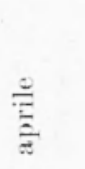 & $=$ & $=$ & 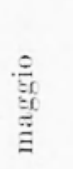 & $=$ \\
\hline & 10 & ai & $\stackrel{\sim}{\sim}$ & $\stackrel{10}{9}$ & $\stackrel{\text { I }}{-}$ & $\cong$ & $=$ & $\cong$ & $\stackrel{\circ}{\leftrightarrow}$ & $\theta$ & 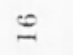 \\
\hline z & $\vec{\Delta}$ & $\stackrel{10}{\circ}$ & $\ddot{\infty}$ & $\stackrel{1}{\infty}$ & $\therefore$ & $\vec{\otimes}$ & $\stackrel{9}{7}$ & $=$ & $\stackrel{\sim}{+}$ & $\dddot{\leftrightarrow}$ & $\mp$ \\
\hline
\end{tabular}




\begin{tabular}{|c|c|c|c|c|c|c|c|c|c|c|c|}
\hline $\begin{array}{l}\stackrel{*}{=} \\
= \\
\|_{\omega}\end{array}$ & $\begin{array}{l}\ddot{0} \\
\dot{0} \\
+\end{array}$ & $\frac{1}{9}$ & $\begin{array}{l}0 \\
0 \\
+\end{array}$ & $\stackrel{a}{a}$ & $\stackrel{\infty}{0}$ & $\begin{array}{l}\ddot{0} \\
0\end{array}$ & $\frac{\infty}{0}$ & 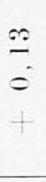 & \pm & $\begin{array}{l}1 \\
0 \\
0\end{array}$ & $\begin{array}{l}0 \\
0 \\
0\end{array}$ \\
\hline$z$ & $\begin{array}{l}5 \\
\infty \\
\infty\end{array}$ & $\begin{array}{l}\ddot{0} \\
\dot{+}\end{array}$ & $\begin{array}{l}= \\
F\end{array}$ & $\begin{array}{l}\overline{1} \\
+\end{array}$ & $\begin{array}{l}9 \\
+\end{array}$ & $\begin{array}{l}10 \\
12 \\
\infty\end{array}$ & $\frac{10}{\infty}$ & $\begin{array}{l}\stackrel{0}{0} \\
+\end{array}$ & $\stackrel{\leftrightarrow}{\dddot{f}}$ & $\begin{array}{l}+\overrightarrow{0} \\
\infty\end{array}$ & $\vec{\theta}$ \\
\hline -i & $>$ & 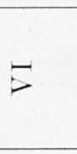 & $>$ & $\bar{D}$ & $\begin{array}{l}\Xi \\
\vdots \\
\vdots\end{array}$ & $>$ & $z$ & $\begin{array}{l}\Xi \\
\overline{1} \\
\overline{1}\end{array}$ & $\vec{z}$ & $>$ & $z$ \\
\hline 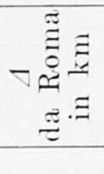 & $\underset{\approx}{\approx}$ & $\stackrel{\Xi}{\circ}$ & $\stackrel{\circ}{\hat{\sigma}}$ & 곡 & $\S$ & $=$ & $\hat{=}$ & $=$ & $=$ & $=$ & $\hat{=}$ \\
\hline 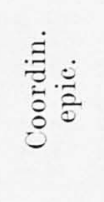 & 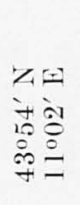 & 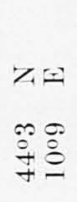 & 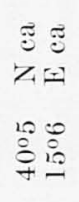 & 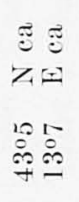 & 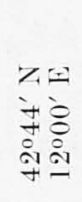 & $\begin{array}{l}= \\
= \\
=\end{array}$ & $\begin{array}{l}= \\
= \\
=\end{array}$ & $\begin{array}{l}= \\
= \\
=\end{array}$ & $\begin{array}{l}= \\
= \\
=\end{array}$ & $\begin{array}{l}= \\
= \\
=\end{array}$ & $\begin{array}{l}= \\
= \\
=\end{array}$ \\
\hline 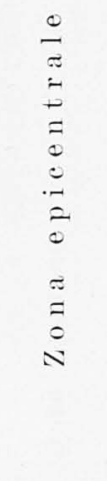 & 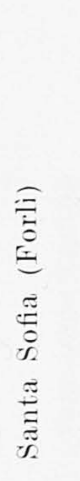 & 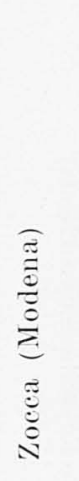 & 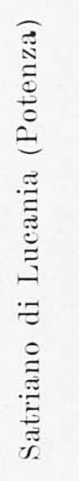 & 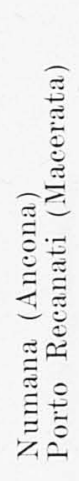 & 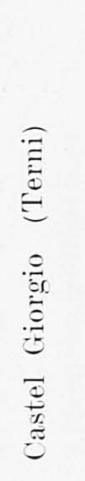 & $\begin{array}{l}= \\
=\end{array}$ & $\begin{array}{l}= \\
=\end{array}$ & $\begin{array}{l}= \\
=\end{array}$ & $=$ & $\begin{array}{l}= \\
= \\
=\end{array}$ & $=$ \\
\hline $\begin{array}{l}\infty \\
\equiv \\
\Rightarrow\end{array}$ & $\begin{array}{l}3 \\
0 \\
0 \\
0 \\
0\end{array}$ & $\begin{array}{l}q \\
\dot{q} \\
\stackrel{1}{0} \\
\stackrel{-}{-}\end{array}$ & $\begin{array}{l}\tilde{J} \\
\pm \\
5\end{array}$ & $\begin{array}{l}3 \\
0 \\
j \\
\text { i } \\
\text { i }\end{array}$ & $\begin{array}{l}0 \\
0 \\
10 \\
0 \\
0\end{array}$ & $\begin{array}{l}\dot{0} \\
0 \\
0 \\
0\end{array}$ & $\begin{array}{l}\overrightarrow{0} \\
\overrightarrow{0} \\
0\end{array}$ & $\begin{array}{l}0 \\
01 \\
0 \\
0\end{array}$ & $\begin{array}{l}0 \\
10 \\
10 \\
0\end{array}$ & $\begin{array}{l}\stackrel{0}{0} \\
0 \\
0 \\
9 \\
-1\end{array}$ & $\begin{array}{l}6 \\
10 \\
\infty \\
\vec{z}\end{array}$ \\
\hline & $\begin{array}{l}1.0 \\
0 \\
0\end{array}$ & $=$ & $=$ & $=$ & $=$ & $=$ & $=$ & $=$ & $=$ & $=$ & $=$ \\
\hline $\begin{array}{l}\pi \\
+ \\
\pi \\
0\end{array}$ & 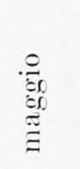 & 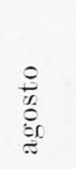 & $\frac{\stackrel{0}{3}}{0}$ & $\begin{array}{l}\stackrel{0}{\vdots} \\
\stackrel{\Xi}{\Xi} \\
\vdots \\
\vdots \\
\Xi\end{array}$ & ڤ્ટ & $=$ & $=$ & $=$ & $=$ & $=$ & $=$ \\
\hline & งิ & to & $\underline{-}$ & $=$ & 0 & $=$ & $=$ & $=$ & $=$ & $=$ & $=$ \\
\hline z & $\stackrel{10}{+}$ & $\stackrel{\bullet}{+}$ & 年 & $\stackrel{\infty}{+}$ & $\stackrel{\circ}{+}$ & 8 & $\bar{E}$ & is & is & 落 & 政 \\
\hline
\end{tabular}




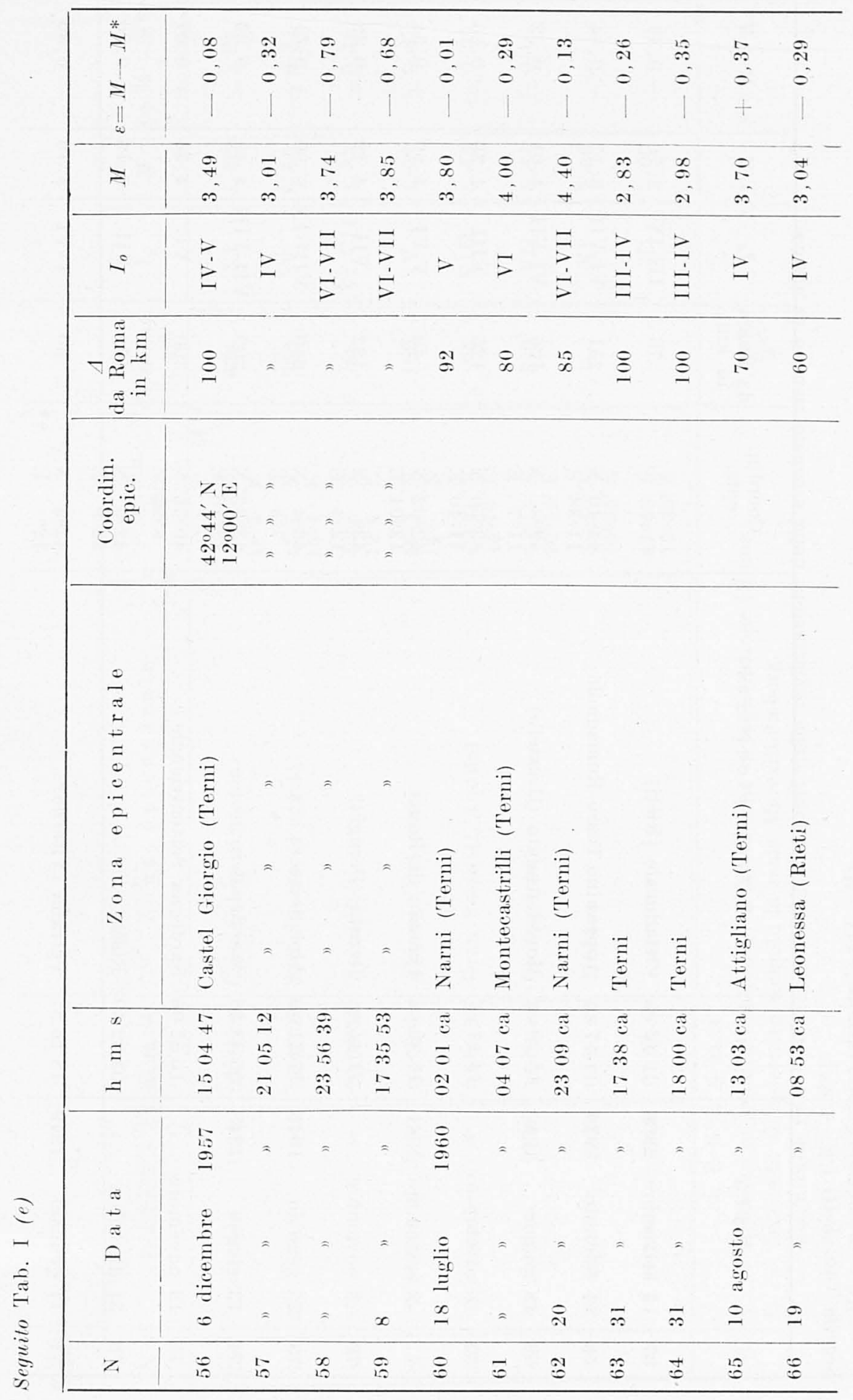




\begin{tabular}{|c|c|c|c|c|c|c|c|c|c|c|c|}
\hline $\begin{array}{l}\stackrel{*}{=} \\
= \\
\|\end{array}$ & $\ddot{0}$ & $\begin{array}{l} \pm \\
0 \\
0 \\
+\end{array}$ & $\begin{array}{l}\therefore \\
0 \\
0\end{array}$ & $\begin{array}{l}\infty \\
\infty \\
0\end{array}$ & $\begin{array}{l}+ \\
0 \\
0 \\
+\end{array}$ & $\stackrel{\infty}{+}$ & $\begin{array}{l}\overline{10} \\
0 \\
+\end{array}$ & $\begin{array}{l}0 \\
0 \\
0\end{array}$ & $\begin{array}{l}5 \\
0 \\
+\end{array}$ & $\begin{array}{l}0 \\
0 \\
+\end{array}$ & $\begin{array}{l}0 \\
\infty \\
0\end{array}$ \\
\hline$=$ & $\begin{array}{l}R \\
\therefore\end{array}$ & $\frac{1}{10}$ & $\begin{array}{l}\overline{0} \\
\dot{+}\end{array}$ & $\begin{array}{l}\stackrel{\theta}{-} \\
+\end{array}$ & $\begin{array}{l}\dot{\theta} \\
+\end{array}$ & $\begin{array}{l}\vec{i} \\
\dot{+}\end{array}$ & 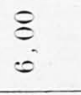 & $\begin{array}{l}\infty \\
+ \\
+\end{array}$ & \begin{tabular}{l}
0 \\
\hdashline \\
+
\end{tabular} & $\stackrel{\circledast}{\because}$ & $\stackrel{\oplus}{\infty}$ \\
\hline- & $\begin{array}{l}\Xi \\
\Xi\end{array}$ & $\begin{array}{l}5 \\
5\end{array}$ & $\begin{array}{l}\overline{5} \\
\overline{5}\end{array}$ & $\Xi$ & 5 & $=$ & $\begin{array}{l}\searrow \\
\Xi \\
\Xi\end{array}$ & $\begin{array}{l}\Xi \\
\Xi \\
\Xi\end{array}$ & $\overline{-}$ & $\equiv$ & $\overline{5}$ \\
\hline 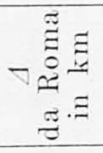 & ${ }_{10}^{10}$ & $\bar{s}$ & $\stackrel{\sim}{\sim}$ & $\stackrel{19}{\mathrm{I}}$ & $\stackrel{\infty}{+}$ & $\stackrel{i}{\stackrel{i}{-}}$ & $\stackrel{0}{0}$ & $\stackrel{\text { ๙ }}{1}$ & $\stackrel{8}{\circ}$ & $\stackrel{R}{R}$ & $\therefore$ \\
\hline$\frac{\dot{\Xi}}{\dot{\Xi}} \cdot \frac{\dot{\Xi}}{0}$ & & 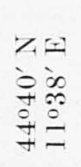 & 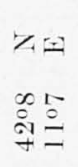 & $\begin{array}{l}71=1 \\
00 \\
100 \\
09 \\
90 \\
7\end{array}$ & 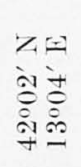 & $\begin{array}{l}Z=1 \\
\stackrel{+}{\circ} \stackrel{\circ}{\rightarrow}\end{array}$ & $\begin{array}{l}Z=1 \\
+10 \\
\dot{0} 0\end{array}$ & 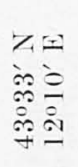 & $\begin{array}{l}z=1 \\
000 \\
10 \\
80 \\
9\end{array}$ & $\begin{array}{l}710 \\
100 \\
00 \\
i 1 \\
+1\end{array}$ & $\begin{array}{l}71= \\
\ddot{\circ} \stackrel{\circ}{\circ} \\
\stackrel{9}{+}\end{array}$ \\
\hline 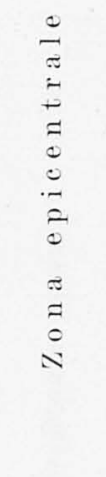 & 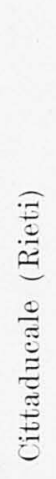 & 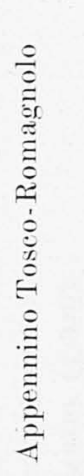 & 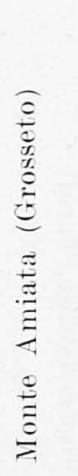 & $=$ & 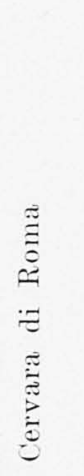 & 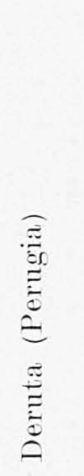 & 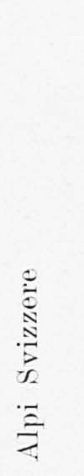 & 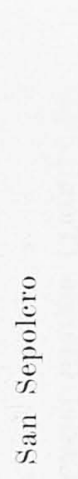 & 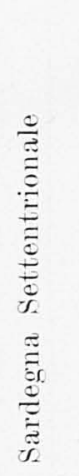 & $\frac{\pi}{2}$ & 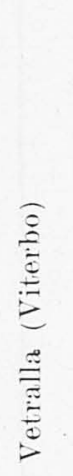 \\
\hline $\begin{array}{l}\infty \\
\Xi \\
\approx\end{array}$ & \begin{tabular}{l}
$\tilde{5}$ \\
\multirow{3}{*}{} \\
0 \\
0
\end{tabular} & $\begin{array}{l}\tilde{J} \\
\underline{z} \\
=\end{array}$ & $\begin{array}{l}\frac{\pi}{5} \\
\frac{0}{10}\end{array}$ & $\begin{array}{l}\tilde{3} \\
\pm \\
\pm\end{array}$ & $\begin{array}{l}\tilde{E} \\
0 \\
\infty \\
\underline{L}\end{array}$ & $\begin{array}{l}\vec{J} \\
\dot{J} \\
\frac{\pi}{1}\end{array}$ & $\begin{array}{l}8 \\
\bar{\infty} \\
=\end{array}$ & $\begin{array}{l}\ddot{\infty} \\
\approx \\
\ddot{0} \\
\ddot{0}\end{array}$ & $\begin{array}{l}\stackrel{0}{0} \\
\text { iv } \\
0 \\
0\end{array}$ & 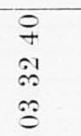 & $\begin{array}{l}10 \\
0 \\
0 \\
0\end{array}$ \\
\hline & $\stackrel{8}{\mathscr{G}}$ & $\stackrel{\mathscr{O}}{\mathscr{O}}$ & $\stackrel{9}{ \pm}$ & $=$ & $\Xi$ & $=$ & $\stackrel{0}{\Xi}$ & $\stackrel{\infty}{ \pm}$ & $=$ & $=$ & $\stackrel{g}{\dot{t}}$ \\
\hline $\begin{array}{l}\pi \\
+ \\
\pi \\
0\end{array}$ & $\frac{\stackrel{D}{\Xi}}{\stackrel{\Xi}{\Xi}}$ & $\begin{array}{l}\frac{0}{3} \\
\frac{0}{6} \\
\frac{0}{0} \\
= \\
=\end{array}$ & 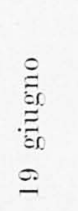 & $\begin{array}{l}0 \\
\frac{0}{0} \\
\frac{1}{ \pm} \\
0 \\
0\end{array}$ & 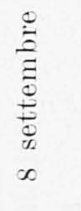 & 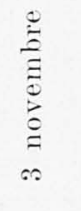 & 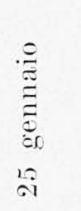 & 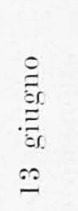 & 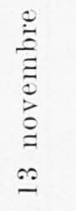 & 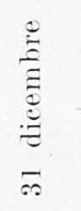 & 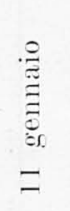 \\
\hline z & 5 & $\infty$ & $\dot{\theta}$ & $R$ & $\pi$ & $i$ & $\stackrel{0}{i}$ & $\underset{⿱}{\mathbb{2}}$ & ${ }_{10}^{10}$ & $\underset{N}{0}$ & 永 \\
\hline
\end{tabular}


Seguilo Tab. 1 (g)

\begin{tabular}{|c|c|c|c|c|c|c|c|c|c|}
\hline$N$ & \multicolumn{2}{|l|}{ I) $a t a$} & ll $\quad$ III $\quad$ s & tona epicentrale & $\begin{array}{l}\text { Coordin. } \\
\text { epice. }\end{array}$ & $\begin{array}{c}1 \\
\text { da Romal } \\
\text { in } k m \text { m }\end{array}$ & $I_{0}$ & $.1 I$ & $\varepsilon=. I I-. I I^{*}$ \\
\hline $7 s$ & 8 ottohre & $1 ! 4 ! !$ & $03018 \times a$ & Caltagirone (Catania) & $\begin{array}{ll}3603 & N \\
1+0.5 & \mathrm{E}\end{array}$ & 630 & $\mathrm{VI}$ & 5,50 & $+1,21 ?$ \\
\hline 79 & 24 & $"$ & $17+800$ & San Severo (Foggia) & $\begin{array}{ll}+107 & \mathrm{X} \\
1504 & \mathrm{E}\end{array}$ & 260 & 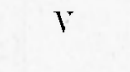 & 3,70 & $-0,11$ \\
\hline 80 & 27 & $"$ & 200834 & Terni-Rieti & $\begin{array}{ll}+2^{\circ} 6 & N \\
12^{\circ} 6 & \mathrm{l}\end{array}$ & 88 & VI-VII & 4,35 & -0.18 \\
\hline 81 & 7 marzo & 1950 & $0+0550$ & Alamo (Pescara) & $\begin{array}{ll}+203 & N \\
1+0 & K\end{array}$ & 160 & $V$ & 4,68 & $+0,87$ \\
\hline 82 & 5 setiembre & $n$ & $0+0857$ & Gran Sasso (l'Aquila) & $\begin{array}{l}+2031^{\prime} \times \\
13019 \\
1\end{array}$ & 95 & VIII & 5,45 & $+0,20$ \\
\hline 83 & 15 ottobre & 1939 & 150500 & Garfagnana & $\begin{array}{l}+4^{\circ} \mid t^{\prime} N \\
10^{\circ} \mid 2^{\prime} \mathrm{l}\end{array}$ & 319 & VII & 5,41 & $+0,64$ \\
\hline 84 & 28 dicembre & I9OS & 0.52027 & Messina & $\begin{array}{l}38^{\circ} 10^{\prime} N \\
15^{\circ} 35^{\prime}\end{array}$ & - & XI & 7,50 & 一 \\
\hline 8.5 & 13 gennaio & 1915 & $0752+3$ & A vezzano & $\begin{array}{l}+1^{\circ} 59^{\prime} \\
13^{\circ} 36^{\prime}\end{array}$ & - & $\mathrm{X}$ & 7,00 & - \\
\hline & $\begin{array}{l}1=\text { distany } \\
I_{0}=\text { Intensi } \\
y=\text { magnit } \\
\varepsilon=\text { searti } \\
y^{*}=\text { magnit. }\end{array}$ & 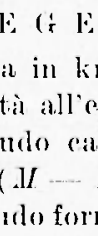 & 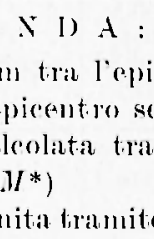 & $\begin{array}{l}\text { centro e las stabione di registraz } \\
\text { meondo la scala Mercalli-sieberg. } \\
\text { mite i dati rieavati dalle regist } \\
\text { e la } f\left(I_{0}, M\right) \text {. }\end{array}$ & $\begin{array}{l}\text { ma). } \\
\text { lella stazion }\end{array}$ & sismica di & Romil. & & \\
\hline
\end{tabular}




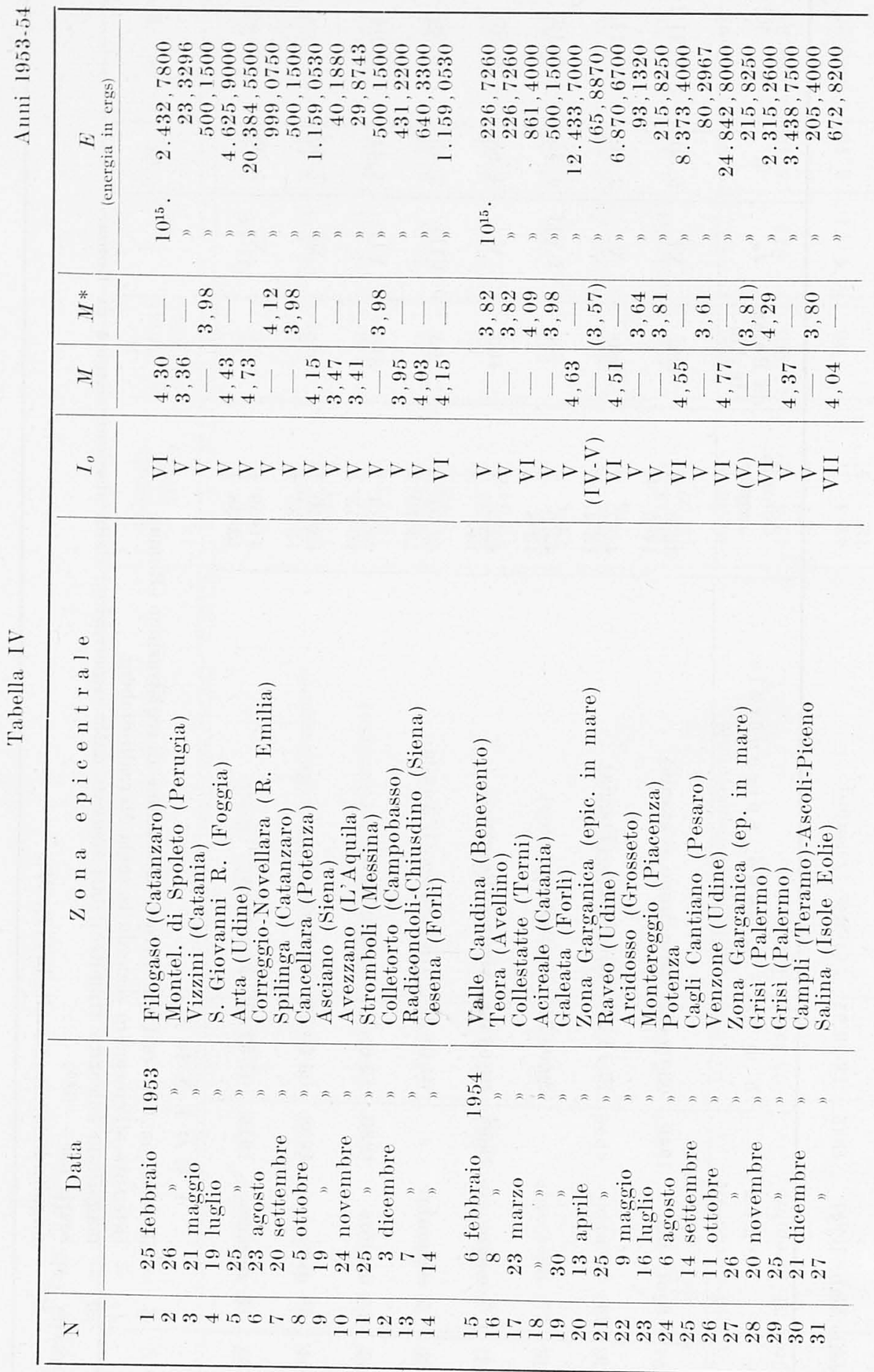




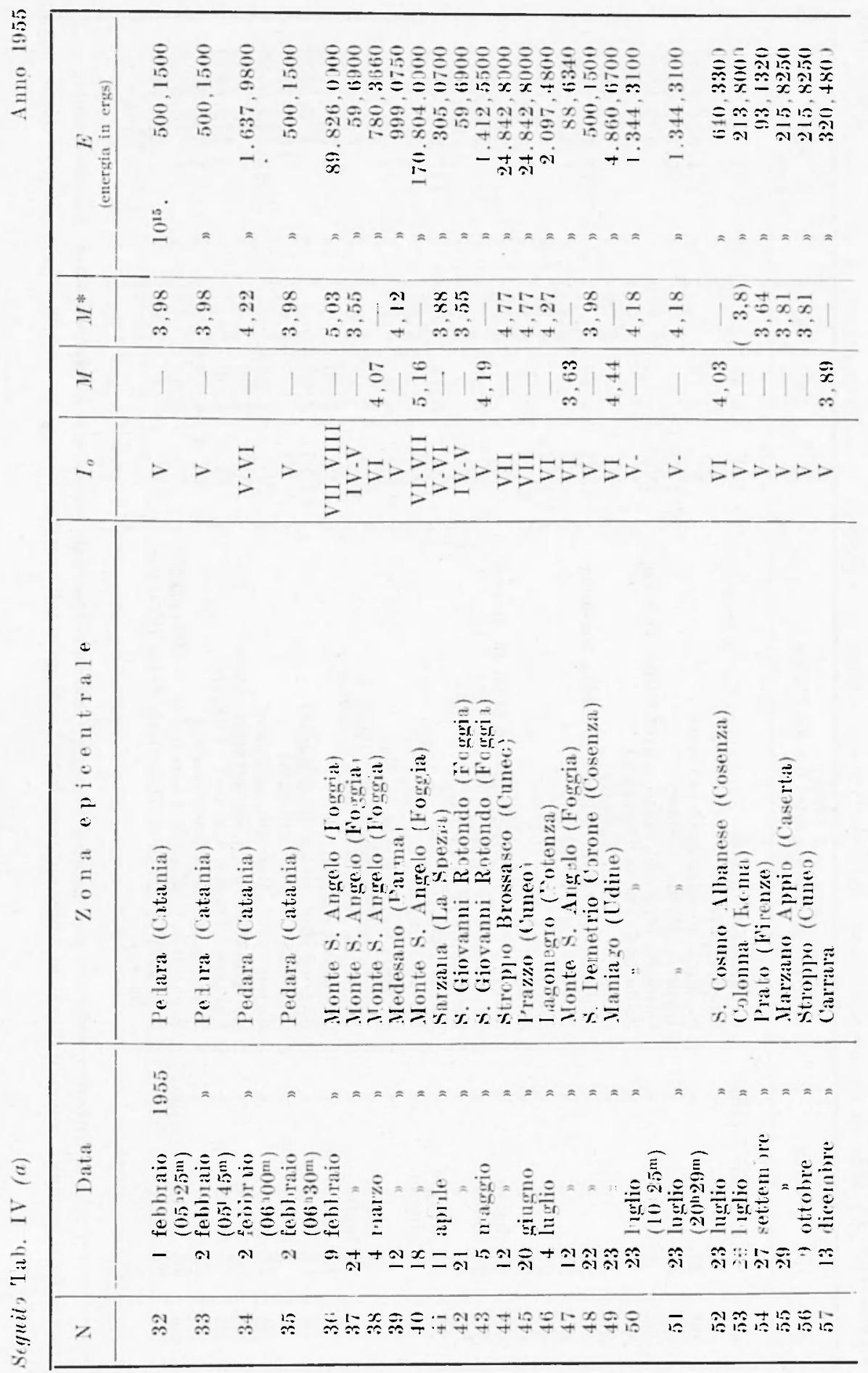




\begin{tabular}{|c|c|c|c|c|c|c|c|c|c|}
\hline 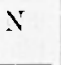 & \multicolumn{3}{|c|}{ I) ata } & \%ona epicentrale & $I_{0}$ &.$M$ &.$l^{*}$ & \multicolumn{2}{|c|}{$\begin{array}{c}\qquad \\
\text { (enlergia in erks) }\end{array}$} \\
\hline 58 & 9 & gentmilo & 1956 & (Grassano (Matera) & VII & $4,+1$ & - & $10^{15}$ & $+.190(1,8200$ \\
\hline $5 !$ & 15 & $"$ & n & Grisì (Palcrumo) & Y & 3.79 & ...- & " & $195,+800$ \\
\hline 60 & 27 & $"$ & $"$ & $\therefore$. Iartino di l'inita (Cosenza) & r & 4. Is & -..- & $n$ & $1.34+.31010$ \\
\hline $6 I^{*}$ & 31 & $"$ & 11 & Istria - Monte Nevoso & VII-VIII & - & $\overline{5}, 0 \overline{5}$ & " & $99.175,(10000$ \\
\hline 62 & 1 & febbraio & i) & Cosenza (epic. in mare - profondità 215 kinl) & (V) & - & $(3,98)$ & $n$ & 500,1500 \\
\hline 63 & 6 & $"$ & $n$ & Vafferana linea (Catania) & Y & - & 3,98 & $n$ & 500,1500 \\
\hline 64 & 19 & $"$ & $"$ & Hontes. Angelo (Fogria) & I & - & 3,79 & $n$ & $195,+8019$ \\
\hline (ii) & 20 & ” & $n$ & Argenta-Battaglia Terme (Ferrara-ladova) & $V-V I$ & t. (ji) & $\ldots$ & " & $13.727,8000$ \\
\hline 66 & 21 & $"$ & $"$ & Rovereto (Trento) & 1 & . & 4,18 & $n$ & $1.34+3100$ \\
\hline 67 & 22 & $"$ & $"$ & Siena. & VI-VII & - &,+ 36 & $n$ & $3.272,(6+1) 0$ \\
\hline 68 & 23 & ” & $"$ & Siena & I. VI & - & 3,88 & $n$ & 305,0700 \\
\hline 69 & 20 & marzo & $"$ & ('ivezza (Inperia) (probabile epice in mare) & V & - & 3.81 & $n$ & 215,8250 \\
\hline 70 & 26 & ajurila: & 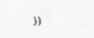 & Pietramala (Fir'enzo) $\left(0 \mathrm{r}^{\mathrm{e}}\left(0 \mathrm{f}^{\mathrm{h}} 00^{\mathrm{m}}\right)\right.$ & VI & 3,88 & - & " & 305,07010 \\
\hline 71 & 26 & 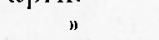 & $"$ & l'ietramala (Firenze) (ore $\left.07^{\mathrm{H}} 13^{\mathrm{m}}\right){ }^{\prime}$ & V. & - & 3,64 & $n$ & 93,1320 \\
\hline 72 & 26 & maggio & $"$ & Santa Sofia (Forli) & lil & 5.13 & $\ldots$ & $"$ & $1+7.20(i 6,0000)$ \\
\hline 73 & 1 & gillogllo & $"$ & Sinvie (Cuneo) & VI & $\div$ &,+ 29 & $"$ & $2.315,2000$ \\
\hline $7+$ & l & $n$ & $"$ & Saluzzo (('uneo) & $(I Y-V)$ & - & $(3,57)$ & $"$ & 6.5. 8570 \\
\hline 75 & 3 & $"$ & $"$ & Rocea S. Casciano (Forli) & lil & 3,66 & + & $n$ & 102,8030 \\
\hline 76 & 23 & lugrlio & $"$ & Monte S. Angelo (Fogreria) & 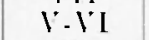 & 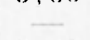 & 4,113 & $"$ & $(i+0,3300$ \\
\hline 77 & 16 & angosto & $"$ & ('ittareale (Rieti) & $Y$ & 3,89 & $\ldots$ & $"$ & 320,4800 \\
\hline 78 & 17 & 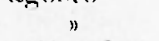 & $"$ & $\therefore$ (Giovamni Rotondo (Fogrgia) & VI.YII & 3,68 & - & $"$ & 113,5000 \\
\hline 79 & l & settembre & $"$ & Monte s. Angelo (Fogria) & V. II & &,+ 013 & $n$ & 640,3300 \\
\hline 80 & 22 & " & $"$ & S. Giovanni Rotondo (Forgia) & VI & 4,63 & - & " & $12 .+33,9000$ \\
\hline 81 & $\overline{3}$ & ottobre & $"$ & Caramanico-Toceo Casauria (l'escara) & $Y$ & 3,20 & $\ldots$ & $"$ & 10,5780 \\
\hline 82 & $\tilde{5}$ & novembre & $"$ & Paluzza. Trepjoo ('arnieo (Ldine) & li &,+ 36 & $\ldots$ & $n$ & $3.272,(i+100$ \\
\hline 83 & 25 & " & $"$ & Hacchiagodena (Campobasso) & VI &,+ 20 & - & $"$ & $1.483,9000$ \\
\hline 84 & 26 & $"$ & $"$ & Amandola-Caldarola (Ascoli-Pic. - Macerata) & v & 3,51 & - & $"$ & $+8,9780$ \\
\hline 85 & 29 & $"$ & $"$ & $\begin{array}{l}\text { Angelo in Grotte-Macchiagodena (Campo- } \\
\text { basso) }\end{array}$ & l' & - & 3,82 & $"$ & 226,7260 \\
\hline
\end{tabular}

(*) Liepicentro di questo sisma è fuori del confine italiano ma date le notevoli ripereussioni macrosismiche estesesi a molti centri italiani, lo abbiano incluso nel computo dellenergiat. 


\begin{tabular}{|c|c|c|c|c|c|c|c|c|c|}
\hline $\mathrm{N}$ & \multicolumn{3}{|c|}{ Dala } & \%olla cpicentrale & \multirow{2}{*}{$\frac{I_{0}}{I . I I}$} & \multirow{2}{*}{$\frac{M}{4.03}$} & \multirow{2}{*}{$\frac{.1 \Gamma^{*}}{\ldots .}$} & \multicolumn{2}{|c|}{$\begin{array}{c}E \\
\text { (energial in erks) }\end{array}$} \\
\hline 86 & 25 & gentraio & 1957 & l'izzoli (L'A (yuila) & & & & $10^{15}$ & 640,3300 \\
\hline 87 & 10 & fohbraio & " & Tolmezzo (Uine) & Y & $\cdots$ & 4,18 & $"$ & $1.344,3100$ \\
\hline 88 & 18 & $"$ & $"$ & Paluzza (lidine) & $y$ & - & 4.18 & $"$ & $1.344,3100$ \\
\hline 89 & 23 & $"$ & $"$ & S. Giovamini Rotonolo (Fogrgia) & $Y$ & - & 3,79 & $"$ & $195,+800$ \\
\hline 90 & 3 & mar\%o & " & Zafferana litucal (C'atiania) & R.II & - & 4,22 & $n$ & $1.037,0700$ \\
\hline 91 & 8 & $"$ & $"$ & Ragusa & V & - & 3,81 & 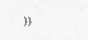 & 215,8250 \\
\hline 92 & r & $"$ & ” & San Reno-Tagria (I mperia) & $y$ & $\ldots$ & 3,81 & $"$ & 215,8250 \\
\hline 93 & 12 & $"$ & $"$ & Norcia (Perugia) & $\mathrm{VI}$ & 4,33 & - & $"$ & $2.821,6250$ \\
\hline 94 & 13 & $n$ & $"$ & Amalrice (Rieti) - ('ascia (Perugia) & $V$ & 3,59 & - & $"$ & 72,7283 \\
\hline 95 & 11 & aprile & $"$ & Potrella Sallo-Vareo Sah. (Rieti) & 11 & 3,95 & - & $"$ & 431,2200 \\
\hline 96 & 17 & (1) & $n$ & S. Soffa (Forli) lore $033^{\circ} 22^{\mathrm{m}}$ ] & III & 4,44 & 一 & 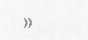 & $4.860,6760$ \\
\hline 97 & 17 & $"$ & $n$ & 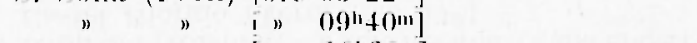 & VII & 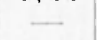 & 4,44 & $"$ & $4.860,6700$ \\
\hline 98 & 17 & $"$ & n & $" \quad\left[n \quad 10^{\mathrm{n}} 30^{\mathrm{m}}\right]$ & $12-1$ &..- & 3,88 & $"$ & 305,0700 \\
\hline 99 & 17 & $"$ & ” & $" \quad\left[177_{50}\left(m^{m}\right]\right.$ & 15 & - & 3,64 & $"$ & 93,1320 \\
\hline 100 & 17 & $"$ & " & $n \quad\left|18^{11} 10^{m+1}\right|$ & IV & - & 3,64 & $"$ & 93,1320 \\
\hline 101 & 18 & $"$ & " & $n \quad\left[07 \mathrm{n}_{30 \mathrm{~m}}\right]$ & III & - & 3,16 & $"$ & 8,$6 ; 796$ \\
\hline 102 & 18 & $"$ & $"$ & $n \quad\left[12^{11} 30 \cdots\right]$ & IV & - & 3,64 & $"$ & 93,1320 \\
\hline 103 & 19 & $n$ & $n$ & $" \quad\left[04^{h} 20 m\right]$ & IV & - & 3,64 & $n$ & 93,1320 \\
\hline 104 & 19 & $n$ & $n$ & $n \quad\left[22^{\mathrm{H}} 15^{\mathrm{m}}\right]$ & IV & - & 3,64 & $"$ & 93,1320 \\
\hline 105 & 20 & $"$ & $"$ & {$\left[\begin{array}{ll}0.4^{11} & 0 . a\end{array}\right]$} & IV & - & 3,64 & $"$ & 93,1320 \\
\hline 106 & 21 & $"$ & $"$ & {$[(0) \mid \div(5) \cdots$} & II & $\cdots$ & 3,64 & $"$ & 93,1320 \\
\hline 107 & 27 & $"$ & $"$ & $\Rightarrow \quad "\left[22^{\prime \prime} 17^{m}\right]$ & II & - & 3,64 & $"$ & 93,1320 \\
\hline 108 & 30 & " & $"$ & l'ieve s. Stefano (Alezzo) & VI & 4,12 & -1 & $"$ & 999,0750 \\
\hline 109 & 3 & margrio & $"$ & Samt Ilario (Polenza) & V.VI & - & 4,013 & $"$ & 640,3300 \\
\hline 110 & 3 & $"$ & $"$ & I aluria (Polenzab) & $1 V-1$ & - & 3,55 & $"$ & $59,(i 900)$ \\
\hline 111 & 6 & $n$ & $n$ & Naso (Messina) & V & 3,86 & - & $n$ & 276,3120 \\
\hline 112 & 11 & $”$ & ” & $\begin{array}{l}\text { Sambuce di Ficilia (Agrigento) - S. Margherila } \\
\text { Bolices }\end{array}$ & $\dot{V}$ & - & 3,81 & $"$ & 215,8250 \\
\hline 113 & 11 & ” & $"$ & Scriacea (A rrigrento) & 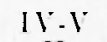 & - & 3,57 & $n$ & $(65,8872$ \\
\hline 114 & 16 & $"$ & $"$ & Force (Ascoli Piceno) & I & 3,77 & $\cdots-$ & $n$ & 177,0920 \\
\hline 115 & 21 & $”$ & $"$ & Palermos [epice in matre] & VIII* & 5,31 & - & $"$ & $358.592,00000$ \\
\hline
\end{tabular}




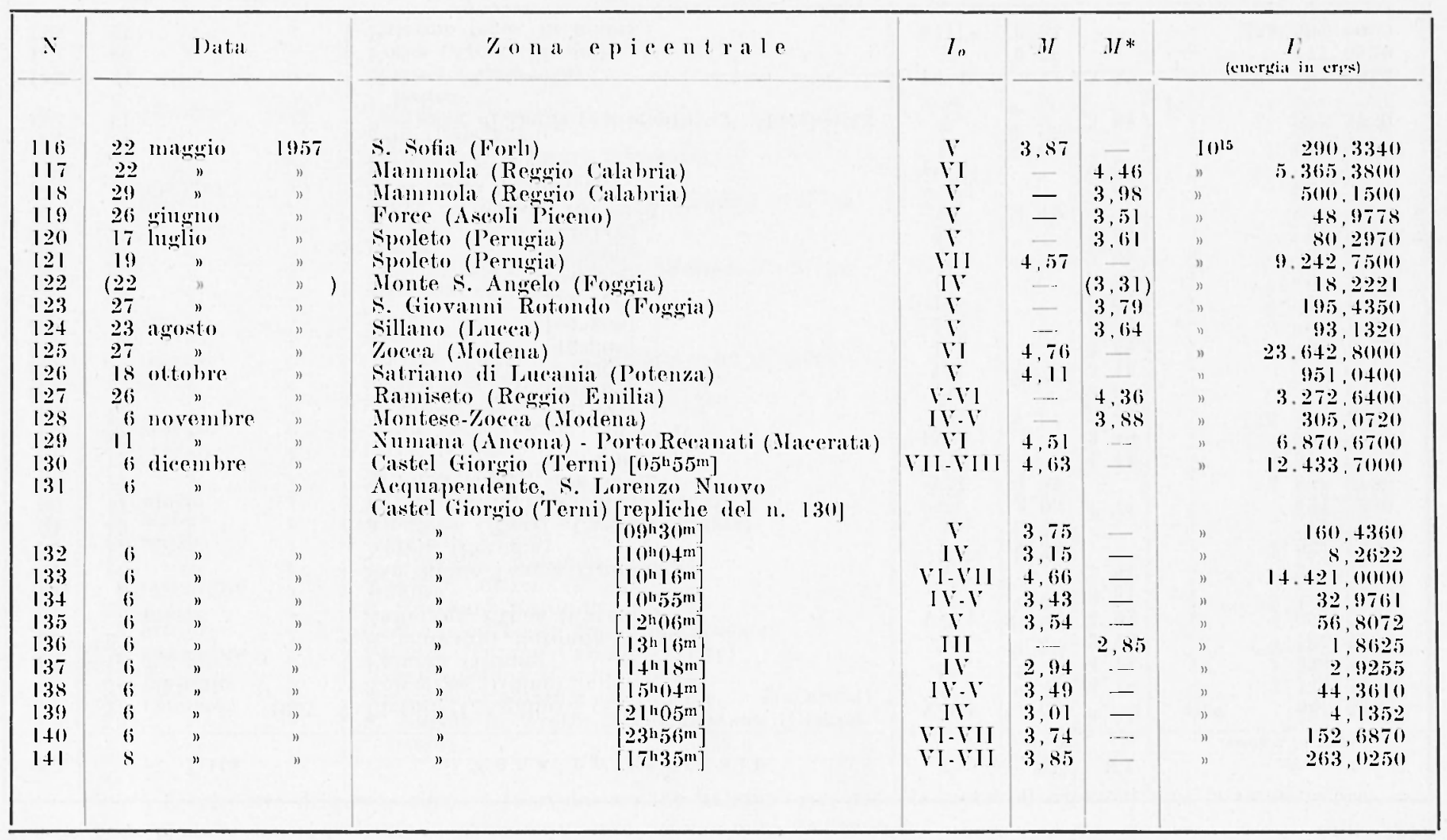


Tahella ${ }^{+}$

\begin{tabular}{|c|c|c|c|c|c|c|c|c|c|c|c|c|c|}
\hline \multirow{2}{*}{$\ln 110$} & \multicolumn{11}{|c|}{ Numero di terremoti di $I_{o}$ (gradi della seala Mercalli-Sieberg) } & \multirow{2}{*}{$\begin{array}{l}\text { N. } \\
\text { anninale } \\
\text { di terr. } \\
\text { sturl. }\end{array}$} & \multirow{2}{*}{$\begin{array}{c}\text { Energia annuale calcolata } \\
\text { in ergs }\end{array}$} \\
\hline & iI & III & IV & $\mathrm{IV}-\mathrm{V}$ & $\mathrm{v}$ & $\mathrm{v}-\mathrm{v} \mathbf{1}$ & vi & VI-VII & vil & VII-VIII & VIII & & \\
\hline 1953 & \multirow{5}{*}{$\left(4_{0}\right)$} & \multirow{4}{*}{$(108)$} & \multirow{4}{*}{$(93)$} & \multirow{2}{*}{$\begin{array}{c}(39) \\
1\end{array}$} & 12 & - & 2 & - & - & - & - & $1+$ & $10^{15} \cdot \quad 33425,8029$ \\
\hline 1954 & & & & & 10 & - & 5 & - & 1 & - & - & 17 & 61638,7677 \\
\hline 1955 & & & & 2 & 13 & 2 & 5 & 1 & 2 & 1 & - & 26 & $329005,+170$ \\
\hline 1956 & & & & 1 & 12 & $t$ & 5 & 2 & 3 & 1 & - & 28 & $\Rightarrow \quad 29+306,5490$ \\
\hline 1957 & & 2 & 12 & (5) & 18 & + & 6 & 3 & 3 & 1 & $1 *$ & 56 & $" \quad 459185,0577$ \\
\hline $\begin{array}{l}\text { Nedi ter- } \\
\text { remoti }\end{array}$ & +0 & 110 & 105 & 49 & 65 & 10 & 23 & (i) & 9 & 3 & 1 & $1+1 \rightarrow$ & 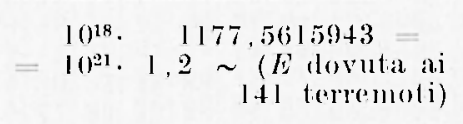 \\
\hline $\begin{array}{l}\text { E(toori- } \\
\text { che per } \\
\text { tratii) }\end{array}$ & $\frac{0.01 \cdot}{1011^{18}}$ & 0,2 & $\begin{array}{l}2.1 \\
10^{18}\end{array}$ & $\begin{array}{l}3,3 \cdot \\
10^{18}\end{array}$ & $\begin{array}{l}14,2 \cdot \\
10^{18}\end{array}$ & $\begin{array}{l}7.2 \\
1()^{18}\end{array}$ & $\begin{aligned} 54,0 \\
10^{18}\end{aligned}$ & $\begin{array}{l}46,4 \cdot \\
10^{18}\end{array}$ & $\frac{229,2 \cdot}{10^{18}}$ & $\begin{array}{l}300,7 \\
10^{18}\end{array}$ & $\begin{array}{l}+17,8.8 \\
1011^{18}\end{array}$ & $\longrightarrow$ & 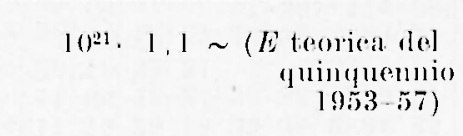 \\
\hline & $\begin{array}{r}(*) \\
\text { registraz } \\
\text { come t }\end{array}$ & $\begin{array}{l}\text { Quest } \\
\text { xioni h } \\
\text { tale lo }\end{array}$ & , b & $\begin{array}{l}\text { moto } \\
\text { ato } \\
\text { an }\end{array}$ & $\begin{array}{l}\text { (puc } \\
\text { valor } \\
\text { irlera }\end{array}$ & $\begin{array}{l}\text { del } \\
\text { dellat } \\
\text {. } 11 \text {. }\end{array}$ & $\begin{array}{l}21 \text { ma } \\
\text { magnit } \\
\text { alo epi }\end{array}$ & $\begin{array}{l}\text { ggio } 19 \\
\text { undo di : } \\
\text { icentro }\end{array}$ & $\begin{array}{l}957 \text { sen } \\
5,31 . A \\
\text { infatti }\end{array}$ & $\begin{array}{l}\text { o a } P \\
\text { e valore } \\
\text { trova }\end{array}$ & $\begin{array}{l}\text { mo } \\
\text { i } 1 / \text { co } \\
\text { mare, }\end{array}$ & $\begin{array}{l}\text { IV.V, n } \\
\text { ponderel } \\
\text { me risul }\end{array}$ & $\begin{array}{l}\text { a che in realtin dalle } \\
\text { be un VIII allepicentro } \\
\text { a dai dati forniti hal- }\end{array}$ \\
\hline
\end{tabular}




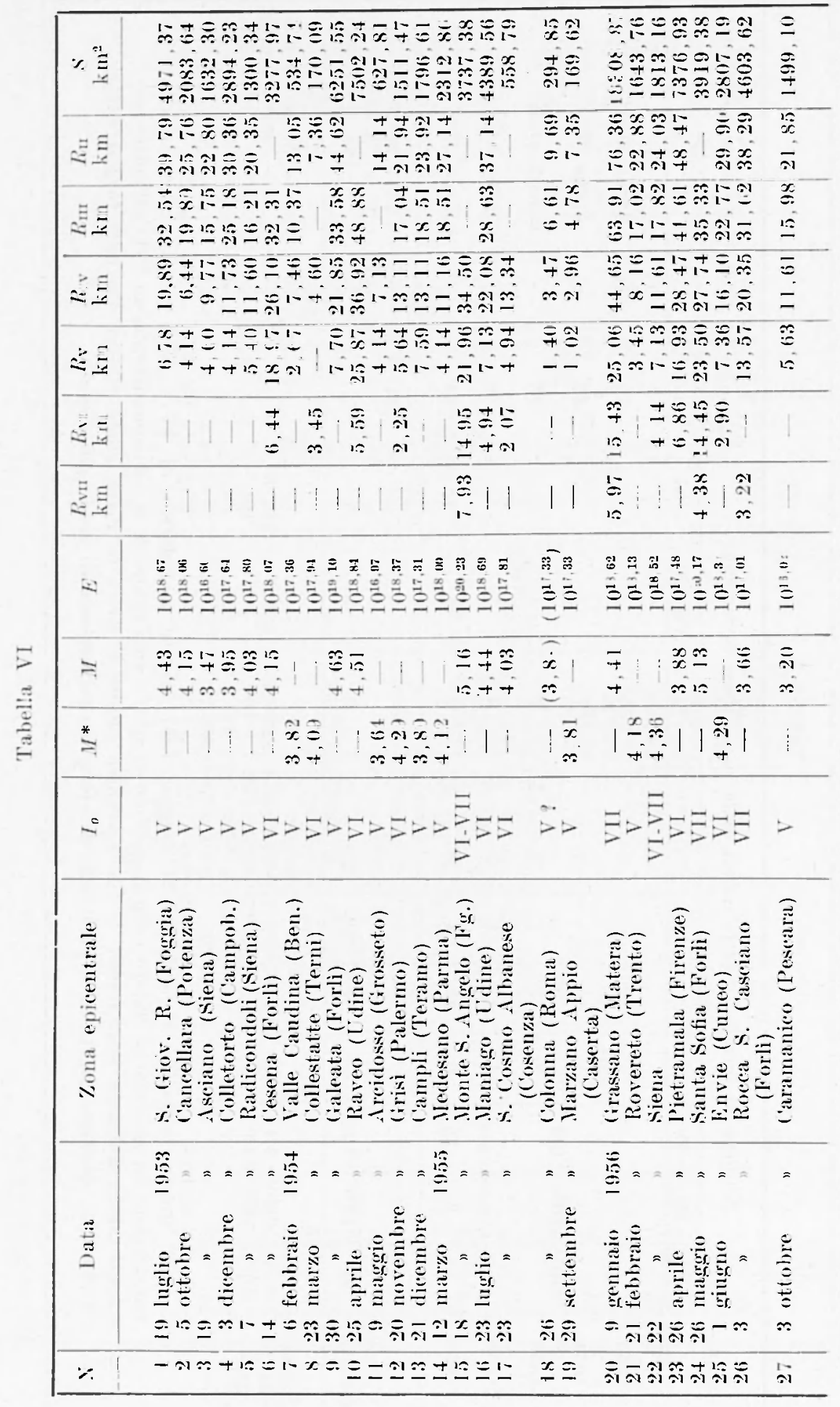




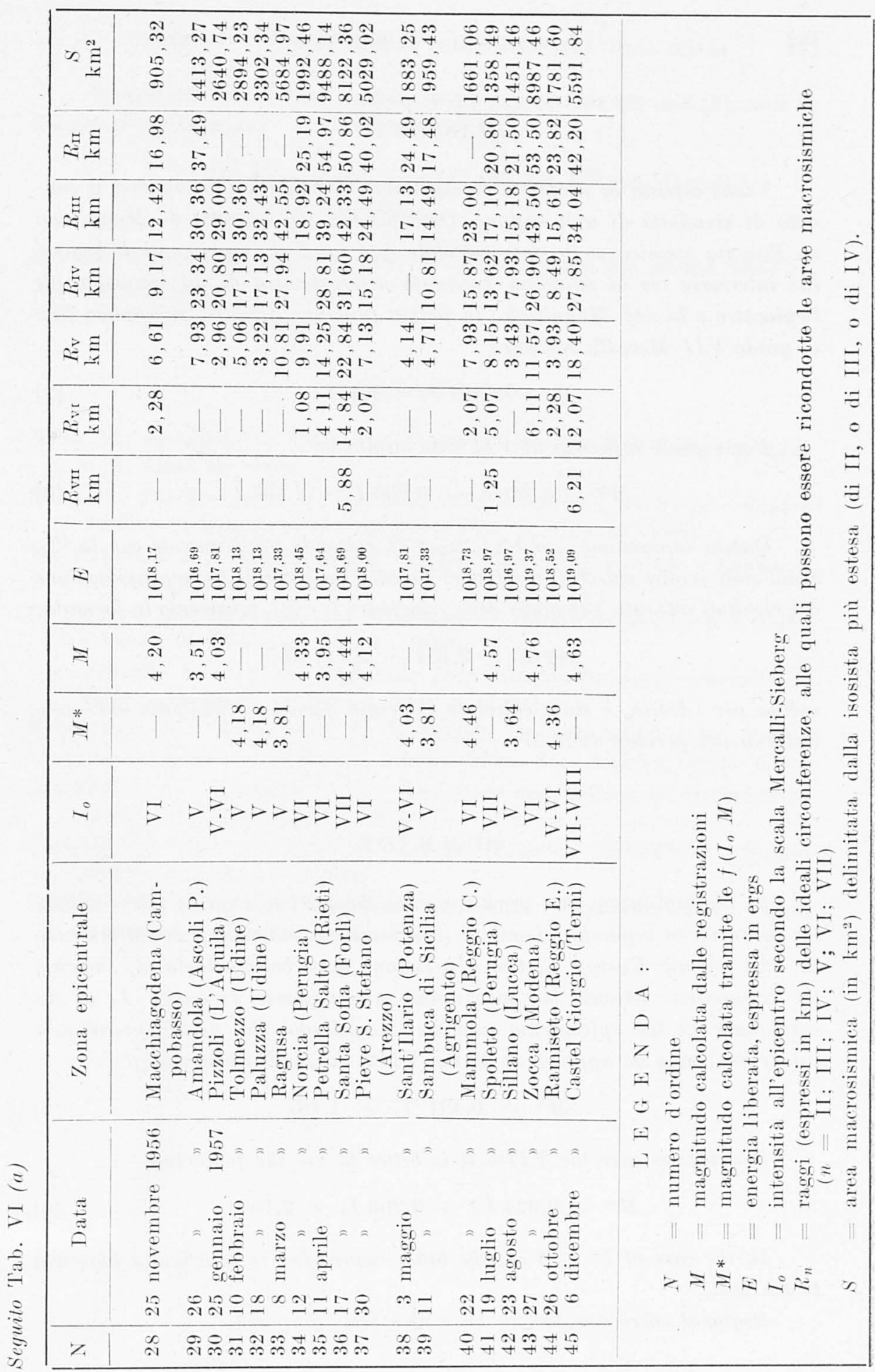




\section{RIASE (NAT)}

Viene espostu la necessita di esprimere in termini piu rigorosi il concrtto di sismicita di una regione. Orientandosi sul concetto di Mugnitudo ed Energia sismica sono state calcolate due funzioni esprimenti il legame che intercorre tra la massima Intensitic macrosismica di un terremoto allepicentro e la sua .Hagnitudo: la prima funzione, lineare, si applica fino al grado VII .Hercalli-Siebery

$$
I^{*}=0,481 I_{0}+1,407 .
$$

P'er i gradi superiori al VII vale meglio la:

$$
I^{*}=0,02+I_{0,2}+0,206 I_{o}+2,157 .
$$

Questa espressione, per la $I_{\Delta} \leqslant V I I$ coincide maticamente con la [2]. Sono stati inoltre calcolati dei fattori correttivi regionali. Come applicuzione dei risultati ottenuti, valendosi delle relazion $i[2]$ [3], attruverso la formula:

$$
\log E=9,15 t+2,1+7 M
$$

valida per l'Italia, ì stata calcolata l'energia sismica stiluppata sul suolo italiano nel periodo 19:5.3-5\%.

\section{$S U M+R)^{\circ}$}

At the beginning, this work concerns the need to express more strictly the meaning of regional seismicity. Successively, starting from Magnitudo II and seismic Energ!y E, two expressions have been calculated, showing the connection between the maximum macroseismie Intensity $I_{n}$ of an earthquake at the epicentrum and its Magnitudo: the first expression, " linear one, may be applied up to the FIIth Inerealli-Sieberg degree:

$$
I^{*}=0,+81 I_{0}+1,407 .
$$

For degrees over the VIIth it is better to use the following:

$$
I^{*}=0,024 I_{0}^{2}+0,206 I_{0}+2,15 \overline{0} \text {. }
$$

In the case of $I_{0} \leqslant V I I$, the latter capression is pretically identical to the first.

Regional corrective factors have also been proposed. 
As application of these studies, using expressions [2] and [3], and by means of the formula:

$$
\begin{aligned}
& \log E=9,151+2,147 \quad U \text { (Di Filippo-Marcelli) } \\
& \text { (valid for Italy) }
\end{aligned}
$$

the total seismic Energy developed in Italy, during the period 19.53-.57, has been calculated.

\section{BIBLIOGRAFIA}

Bßтн M., Seismicity of Europe. A Progress report, I.U.G.G. Monographio n. 1, (Janvier 1960).

BåTu M., The Energy of Seismic Body Waves and Surface Waves "Contributions in Geophysics in Honor of Beno Gutenlberg" 1958.

De Panfilis M., Attività sismica in Italia dal 1953 al 19.5\%. "Annali di Geofisica ", XII, 1, (1959).

Di Filippo D. - Marcertu L., Magnitudo ed energia dei terremoti in Italia "Annali di Geofisica" II I, 3, (1950).

Galanopoulos A. G., On Magnitudo Determination by using maeroseismic Data. "Annali di Geofisica", XIV, 3, (1961).

Gutenberg B. - Ricirper C. F., Earthquake Magnitude, Intensity, Energy and Acceleration. (II Paper). "Bull. Seism. Soc. Amer. ", Aprile 1956).

Gutenberg B. - Ricuter C. F., "Magnitude and Energy of Earthruakes" Annali di Geofisica, IX, 1, (1956).

KaRNik Vit., Epicentre Maps for Europe ( $\left.I_{0} \geqslant \mathrm{VI}, 1901-1955\right)$. "Studia geopl. et geod. ", 5 (1961).

Ricnter C. F., An Instrumental Earthquake Magnitude Scale. "Bull. Seismol. Soc. Amer." (Genn. 1935). 